\title{
Global existence of weak solution for the compressible Navier-Stokes-Poisson system with density-dependent viscosity
}

Meiying Cui ${ }^{1,2^{*}}$ (D) and Wenjing Song ${ }^{1}$

"Correspondence:

mycui2004@163.com

'School of Mathematics and CNS,

Northwest University, Xi'an, China

${ }^{2}$ School of Energy Engineering,

Yulin University, Yulin, China

\begin{abstract}
In this paper, we are concerned with the existence of global weak solutions to the compressible Navier-Stokes-Poisson equations with the non-flat doping profile when the viscosity coefficients are density-dependent, the data are large and spherically symmetric, and we focus on the case where those coefficients vanish in vacuum. We construct a suitable approximate system and consider it in annular regions between two balls. The global solutions are obtained as limits of such approximate solutions. Our proofs are mainly based on the energy and entropy estimates.
\end{abstract}

Keywords: Compressible Navier-Stokes-Poisson equations; Density-dependent; Spherically symmetric

\section{Introduction}

The dynamics of charged particles of one carrier type (e.g., electrons) can be described by the compressible Navier-Stokes-Poisson equations:

$$
\left\{\begin{array}{l}
\rho_{t}+\operatorname{div}(\rho U)=0, \\
(\rho U)_{t}+\operatorname{div}(\rho U \otimes U)+\nabla P(\rho)-\operatorname{div}(h(\rho) D(U))-\nabla(g(\rho) \operatorname{div} U)=\rho \nabla \Phi, \\
\Delta \Phi=\rho-b,
\end{array}\right.
$$

where $(x, t) \in \Omega \times(0, \infty), \rho=\rho(x, t)$ and $u=u(x, t)$ represent the density and velocity functions of the electrons, respectively, at position $x \in \Omega \subset R^{N}(N=2,3)$ and time $t \geq 0$; $P(\rho)=\rho^{\gamma}(\gamma>1)$ denotes the pressure, $D(U)=\frac{1}{2}\left(\nabla U+\nabla U^{\top}\right)$ is the stress tensor, $h(\rho)$ and $g(\rho)$ are the Lamé viscosity coefficients satisfying

$$
h(\rho)>0, \quad h(\rho)+N g(\rho)>0 .
$$

The function $b=b(x)$ is the doping profile for the ions. We assume that $b$ is a smooth function satisfying

$$
b(x)>0, \quad \lim _{|x| \rightarrow+\infty} b(x)=\bar{b}>0 .
$$

(c) The Author(s) 2019. This article is distributed under the terms of the Creative Commons Attribution 4.0 International License (http://creativecommons.org/licenses/by/4.0/), which permits unrestricted use, distribution, and reproduction in any medium, provided you give appropriate credit to the original author(s) and the source, provide a link to the Creative Commons license, and indicate if changes were made. 
The self-consistent electric potential $\Phi=\Phi(x, t)$ is coupled with the density and the doping profile through the Poisson equation.

Such a problem without the Poisson term was studied by many authors; it can be written as

$$
\left\{\begin{array}{l}
\rho_{t}+\operatorname{div}(\rho U)=0 \\
(\rho U)_{t}+\operatorname{div}(\rho U \otimes U)+\nabla P(\rho)-\operatorname{div}(h(\rho) D(U))-\nabla(g(\rho) \operatorname{div} U)=0 .
\end{array}\right.
$$

When the viscosity coefficients $h(\rho)$ and $g(\rho)$ are both constant in (1.4), the onedimensional problem has been studied extensively, see $[14,19,29,30]$ and the references therein. For the multi-dimensional case, the local existence and uniqueness of classical solutions are known $[28,31]$ in the absence of vacuum. The authors of [35] showed both the well- and ill-posedness for the mild solutions of the Navier-Stokes system with dissipation through the generalized Carleson measure spaces of initial data that unify many diverse spaces. Matsumura et al. [24-26] proved global existence of smooth solutions for data close to a non-vacuum equilibrium. In particular, the theory requires that the solution has small oscillations from a uniform non-vacuum state so that the density is strictly away from the vacuum and the gradient of the density remains bounded uniformly in time. The situation becomes more complex for arbitrary data (which may include vacuum states). The major breakthrough is due to Lions [22] (see also Feireisl et al. [11]) who obtained global existence of weak solutions when the exponent $\gamma$ is suitably large, where the only restriction on initial data is that the initial energy is finite, so that the density is allowed to vanish. However, as emphasized in many related papers [15, 16, 20, 21, 36], the regularity, uniqueness, and dynamical behavior of the weak solution for arbitrary initial data remain largely open for compressible Navier-Stokes (CNS) equations with constant coefficients.

By some physical considerations, Liu, Xin, and Yang in [23] introduced the modified CNS equations with density-dependent viscosity coefficients for isentropic fluids. In fact, as presented in [23], while deriving the CNS equations from the Boltzmann equations by the Chapman-Enskog expansions, the viscosity depends on the temperature, and correspondingly on the density for isentropic cases. However, for these more physical models new mathematical challenges are encountered. Degeneration at vacuum occurs because of the dependence of viscosity coefficients on flow density, which makes it very difficult to derive a uniform a priori estimate for the velocity and trace the motion of particle paths near vacuum regions. Moreover, it is not known yet whether or not the vacuum states form for global (weak) solutions to (1.4) even if initial data is far from vacuum. With the help of BD entropy for (1.4) and the compactness results, Bresch et al. have made significant progress on the global existence of weak solutions to the multi-dimensional CNS equations and the 2D shallow water model where either a drag friction or a cold pressure term is involved; we refer to $[1-3,27]$ and references therein. For the spherical symmetric case, $[8,12,13]$ show the global existence of weak solutions of the CNS with density-dependent viscosity, and in [17] the authors have established the regularity for the CNS equations with densitydependent viscosity in $H^{2}$ and $H^{4}$ under certain initial assumptions. Recently, Alexis F. Vasseur and Cheng $\mathrm{Yu}$ [34] have given a breakthrough result of the existence of global weak solutions for 3D compressible Navier-Stokes equations with degenerate viscosity and large initial data, possibly vanishing in vacuum, by deriving a Mellet and Vasseur [27] type inequality for weak solutions. 
For the coupling system of Navier-Stokes and other equation, many authors have given significant results. G.M. Shao and X.J. Chai in [32] proved the unique existence of solutions for the 2D coupling system of Navier-Stokes and electronic equation (i.e., electrohydrodynamics system). Concerning the Navier-Stokes-Poisson (NSP) system, there are also extensive studies about the global existence and dynamical behavior of solutions. For the constant viscosity, Ducomet and Feireisl [5] considered the full NSP equations and proved that, when $\gamma>\frac{3}{2}$, there exists a global-in-time variational weak solution. Ducomet et al. [6] have also proved that there exists a global weak solution to the barotropic compressible NSP equations with nonmonotone pressure, provided that $\gamma>\frac{3}{2}$. Donatelli [7] considered the Cauchy problem for the coupled NSP equations and gave a positive answer to the existence of local and global weak solutions. Zhang and Tan [39], by using the theory of Orlicz spaces, have proved the existence of globally defined finite energy weak solutions. Cai and Tan [4] also proved that the system has a global weak time-periodic solution for the NSP equations in a bounded domain with periodic boundary condition as soon as $\gamma>\frac{5}{3}$ and when the external force is time-periodic. Tan and Wang [33] studied the stability of the steady state of the compressible NSP equations and proved the global existence of solutions near the steady state for the large doping profile.

However, for the case of density-dependent viscosity coefficients, the problem is much more challenge because of the degeneration near the vacuum and the related results are limited. Alexander Zlotnik [40] proved the global-in-time bounds for solutions and studied the large time behavior around a hard core. Under small perturbations, Zhang and Fang [38] obtained the global existence and uniqueness of the weak solution for the spherically symmetric case and without a hard core, also showing that such a system is stable. B. Ducomet et al. [10] considered the Cauchy problem for the Navier-Stoles-Poisson equations of spherically symmetric motions in $R^{3}$, and they proved that the problem admits a global weak solution, provided that the polytropic index $\gamma$ satisfies $\gamma>1$. Duan and Li [9] studied the multi-dimensional compressible NSP system with $\gamma$ law pressure in the simulation of the motion of gaseous stars for $\gamma \in\left(\frac{6}{5}, \frac{4}{3}\right]$.

Inspired by [10] and [12], in the present paper, we consider the global existence of the three-dimensional spherically symmetric solutions of (1.1) with degenerate viscosities; for simplicity, we deal with the case $h(\rho)=\rho, g(\rho)=0$, and take $D(U)=\nabla U$. Our result holds true for general $h(\rho)=\rho^{\alpha}, g(\rho)=(\alpha-1) \rho^{\alpha}$ for some $\alpha>\frac{N-1}{N}(N=2,3)$. We construct a suitable approximate system and, to exclude the singularity at the origin $r=0$, consider the radial symmetric approximate system only on the annular domain. The global existence of classic solutions to such an approximate system can be obtained by the standard arguments. Then we obtain the a priori estimates required in the $L^{1}$ stability analysis. Therefore, by taking a limit, we show that a global spherically symmetric entropy weak solution to (1.1) exists for general initial data with finite entropy for $\gamma \in(1,3)$.

It is different from the situation in [10] where the two viscosity coefficients need to satisfy the relation $\mu(\rho)=\mu_{1} \Psi(\rho), \lambda(\rho)=2 \mu_{1}\left(\rho \Psi^{\prime}(\rho)-\Psi(\rho)\right)$, and $\mu(\rho)$ also has to satisfy another condition $\mu(\rho) \leq C \rho^{\frac{\gamma-1}{2}}$, i.e., the viscosity coefficient $\mu(\rho)$ in [10] needs to be bounded by a function depending on $\rho$ and $\gamma$. However, in this paper, we consider two viscosity coefficients $h(\rho)$ and $g(\rho)$ of the form $h(\rho)=\rho^{\alpha}, g(\rho)=(\alpha-1) \rho^{\alpha}$, and $\alpha$ only needs to satisfy $\alpha>\frac{N-1}{N}(N=2,3)$. 


\section{Preliminaries}

The following lemmas are two standard compactness results and will help us in Sect. 5 get the strong convergence of the solutions.

Lemma 2.1 (Aubin-Lions Lemma) Let $X_{0}, X$ and $X_{1}$ be three Banach spaces with $X_{0} \subseteq$ $X \subseteq X_{1}$. Suppose that $X_{0}$ is compactly embedded in $X$ and that $X$ is continuously embedded in $X_{1}$. For $1 \leq p, q \leq+\infty$, let

$$
W=\left\{u \in L^{p}\left([0, T] ; X_{0}\right) \mid \partial_{t} u \in L^{q}\left([0, T] ; X_{1}\right)\right\} .
$$

(i) If $p<+\infty$, then the embedding of $W$ into $L^{p}([0, T] ; X)$ is compact.

(ii) If $p=+\infty$ and $q>1$, then the embedding of $W$ into $C([0, T] ; X)$ is compact.

Lemma 2.2 (Egorov's uniform convergence theorem) Let $f_{n} \rightarrow f$ a.e. in $\Omega$, a bounded measurable set in $R^{n}$, with $f$ finite a.e. Then for any $\epsilon>0$ there exists a measurable subset $\Omega_{\epsilon} \subset \Omega$ such that $\left|\Omega \backslash \Omega_{\epsilon}\right|<\epsilon$ and $f_{n} \rightarrow f$ uniformly in $\Omega_{\epsilon}$, moreover, if

$$
\begin{aligned}
& f_{n} \rightarrow f \text { a.e. in } \Omega, \\
& f_{n} \in L^{p}(\Omega) \text { and uniformly bounded, for any } 1<p \leq+\infty,
\end{aligned}
$$

then, we have

$$
f_{n} \rightarrow f \quad \text { strongly in } L^{s}(\Omega) \text {, for any } s \in[1, p) .
$$

\section{Main result}

In this paper, we set $h(\rho)=\rho, g(\rho)=0$ and $D(U)=\nabla U$ in $(1.1)_{2}$ for simplicity. Then the isentropic compressible Navier-Stokes-Poisson system (1.1) becomes

$$
\left\{\begin{array}{l}
\rho_{t}+\operatorname{div}(\rho U)=0, \\
(\rho U)_{t}+\operatorname{div}(\rho U \otimes U)+\nabla \rho^{\gamma}-\operatorname{div}(\rho \nabla U)=\rho \nabla \Phi, \quad(x, t) \in \Omega \times(0, \infty), \\
\Delta \Phi=\rho-b .
\end{array}\right.
$$

The initial and boundary conditions are

$$
\begin{aligned}
& \left.(\rho, \rho U, \Phi)\right|_{t=0}=\left(\rho_{0}(x), \mathbf{m}_{0}(x), \Phi_{0}(x)\right), \quad \text { in } \Omega, \\
& m=\rho U=0, \quad \frac{\partial \Phi}{\partial v}=0, \quad \text { on } \partial \Omega,
\end{aligned}
$$

here $v$ is the unit outer normal vector on $\partial \Omega$. Consider a spherically symmetric solution $(\rho, u)$ to (3.1) in a ball $\Omega$ of radius $\mathrm{R}$ centered at the origin in $R^{3}$. Then

$$
\begin{aligned}
& \rho(\mathbf{x}, t)=\rho(r, t), \quad U(\mathbf{x}, t)=u(r, t) \frac{\mathbf{x}}{r}, \quad \Phi(\mathbf{x}, t)=\phi(r, t), \\
& b(\mathbf{x})=b(r), \quad r=|\mathbf{x}|,
\end{aligned}
$$


and system (3.1) is changed to

$$
\left\{\begin{array}{l}
\rho_{t}+(\rho u)_{r}+\frac{2 \rho u}{r}=0 \\
(\rho u)_{t}+\left(\rho u^{2}+\rho^{\gamma}\right)_{r}+\frac{2 \rho u^{2}}{r}-\left(\rho u_{r}\right)_{r}-\rho\left(\frac{2 u}{r}\right)_{r}=\rho \phi_{r} \\
\phi_{r r}+\frac{2}{r} \phi_{r}=\rho-b
\end{array}\right.
$$

for $0<r<R$. The corresponding initial data and boundary conditions are

$$
\begin{aligned}
& \left.(\rho, \rho u, \phi)\right|_{t=0}=\left(\rho_{0}, m_{0}, \phi_{0}\right), \\
& \rho u(0, t)=0, \quad \rho u(R, t)=0, \quad \phi_{r}(0, t)=0, \quad \phi_{r}(R, t)=0 .
\end{aligned}
$$

Moreover, by solving (3.5) 3 , we get $\phi_{r}=\frac{1}{r^{2}} \int_{0}^{r} \rho s^{2} d s-\frac{1}{r^{2}} \int_{0}^{r} b(s) s^{2} d s=: f_{\rho}-f_{b}$.

First we obtain the following usual a priori energy estimates for smooth solutions to (3.1)-(3.3).

Lemma 3.1 If $(\rho, U)$ is a smooth solution to (3.1)-(3.3) and the initial assumption (3.31) is satisfied, then the following inequality holds for $T>0$ :

$$
\int_{\Omega}\left\{\frac{1}{2} \rho U^{2}+\frac{1}{2}|\nabla \Phi|^{2}+\frac{\rho^{\gamma}}{\gamma-1}\right\} d x+\int_{0}^{t} \int_{\Omega} \rho|\nabla U|^{2} d x d t \leq C,
$$

here $C$ is a positive constant.

Proof Multiplying $(3.1)_{1}$ by $\frac{U^{2}}{2},(3.1)_{1}$ by $\frac{\gamma}{\gamma-1}$ and $(3.1)_{2}$ by $U$, then summing up and integrating the resulting formula with respect to $x$ by parts, one can easily get

$$
\frac{d}{d t} \int_{\Omega}\left\{\frac{1}{2} \rho U^{2}+\frac{1}{2}|\nabla \Phi|^{2}+\frac{\rho^{\gamma}}{\gamma-1}\right\} d x+\int_{\Omega} \rho|\nabla U|^{2} d x \leq 0 .
$$

Integrating the above formula with respect to $t$, we obtain

$$
\begin{gathered}
\int_{\Omega}\left\{\frac{1}{2} \rho U^{2}+\frac{1}{2}|\nabla \Phi|^{2}+\frac{\rho^{\gamma}}{\gamma-1}\right\} d x+\int_{0}^{t} \int_{\Omega} \rho|\nabla U|^{2} d x d t \\
\leq \int_{\Omega}\left\{\frac{1}{2} \frac{\mathbf{m}_{0}^{2}}{\rho_{0}}+\frac{1}{2}\left|\nabla \Phi_{0}\right|^{2}+\frac{\rho_{0}^{\gamma}}{\gamma-1}\right\} d x .
\end{gathered}
$$

Integrating by parts yields

$$
\begin{aligned}
\int_{\Omega}\left|\nabla \Phi_{0}\right|^{2} d x & =-\int_{\Omega} \Phi_{0}\left(\rho_{0}-b\right) d x \\
& \leq\left\|\rho_{0}-b\right\|_{L^{p}(\Omega)}\left\|\Phi_{0}\right\|_{L^{p^{\prime}}(\Omega)} \\
& \leq\left\|\rho_{0}-b\right\|_{L^{\beta}(\Omega)}^{\theta}\left\|\rho_{0}-b\right\|_{L^{1}(\Omega)}^{1-\theta}\left\|\Phi_{0}\right\|_{L^{p^{\prime}}(\Omega)},
\end{aligned}
$$

where $\frac{1}{p}+\frac{1}{p^{\prime}}=1, \frac{1}{p}=\frac{\theta}{\beta}+\frac{1-\theta}{1}$.

Then by Hardy-Littlewood-Sobolev imbedding theorem, one has

$$
\left\|\Phi_{0}\right\|_{L^{p^{\prime}(\Omega)}} \leq C(\beta)\left\|\rho_{0}-b\right\|_{L^{\beta}(\Omega)},
$$


here $\frac{1}{\beta}=\frac{1}{p^{\prime}}+\frac{2}{3}$. Hence we have

$$
\int_{\Omega}\left|\nabla \Phi_{0}\right|^{2} d x \leq C(\beta)\left\|\rho_{0}-b\right\|_{L^{\beta}(\Omega)}^{1+\theta}\left\|\rho_{0}-b\right\|_{L^{1}(\Omega)}^{1-\theta} .
$$

It follows from $\frac{1}{\beta}=\frac{1}{p^{\prime}}+\frac{2}{3}, \frac{1}{p}+\frac{1}{p^{\prime}}=1, \frac{1}{p}=\frac{\theta}{\beta}+\frac{1-\theta}{1}$ and $0<\theta<1$ that $\frac{6}{5}<\beta<\frac{3}{2}$, thus $W^{1,2}(\Omega) \hookrightarrow L^{\beta}(\Omega)$.

Under the initial assumption (3.31), we can deduce

$$
\int_{\Omega}\left|\nabla \Phi_{0}\right|^{2} d x \leq C(\beta)\left\|\rho_{0}-b\right\|_{W^{1,2}(\Omega)}^{1+\theta}\left\|\rho_{0}-b\right\|_{L^{1}(\Omega)}^{1-\theta} \leq C
$$

Returning to (3.10), we can obtain the desired basic energy estimate (3.8).

It is also easy to get the following usual a priori energy estimate for smooth solutions to (3.5)-(3.7):

$$
\frac{d}{d t} \int_{0}^{R}\left\{\frac{1}{2}\left(\rho u^{2}+\phi_{r}^{2}\right)+\frac{\rho^{\gamma}}{\gamma-1}\right\} r^{2} d r+\int_{0}^{R} \rho\left(u_{r}^{2} r^{2}+2 u^{2}\right) d r \leq 0
$$

and then

$$
\int_{0}^{R}\left\{\frac{1}{2} \rho u^{2}+\frac{1}{2} \phi_{r}^{2}+\frac{\rho^{\gamma}}{\gamma-1}\right\} r^{2} d r+\int_{0}^{t} \int_{0}^{R} \rho\left(u_{r}^{2} r^{2}+2 u^{2}\right) d r d s \leq C,
$$

where $C$ is a positive constant.

Furthermore, system (3.1) admits an additional a priori estimate, as observed by Bresch, Desjardins, and Lin [3], which reads as follows for three-dimensional spherically symmetric equations (3.5):

Lemma 3.2 If $(\rho, u)$ is a smooth solution to (3.5) with $\rho>0$, then the following inequality holds:

$$
\begin{aligned}
& \frac{d}{d t} \int_{0}^{R}\left\{\frac{1}{2} \rho\left|u+(\log \rho)_{r}\right|^{2}+\frac{1}{2} \phi_{r}^{2}+\frac{\rho^{\gamma}}{\gamma-1}\right\} r^{2} d r+\int_{0}^{R} \frac{4}{\gamma}\left(\left(\rho^{\frac{\gamma}{2}}\right)_{r} r\right)^{2} d r \\
& \quad+\int_{0}^{R}\left\{(\rho-b)^{2}+\rho b\right\} r^{2} d r \leq C,
\end{aligned}
$$

i.e.,

$$
\begin{aligned}
& \frac{d}{d t} \int_{0}^{R}\left\{\frac{1}{2} \rho u^{2}+\rho_{r} u+\left|(\sqrt{\rho})_{r}\right|^{2}+\frac{1}{2} \phi_{r}^{2}+\frac{\rho^{\gamma}}{\gamma-1}\right\} r^{2} d r+\int_{0}^{R} \frac{4}{\gamma}\left(\left(\rho^{\frac{\gamma}{2}}\right)_{r} r\right)^{2} d r \\
& \quad+\int_{0}^{R}\left\{(\rho-b)^{2}+\rho b\right\} r^{2} d r \leq C,
\end{aligned}
$$

where $C$ is a positive constant.

Proof Multiplying equation $(3.5)_{1}$ by $\frac{\left|(\log \rho)_{r}\right|^{2} r^{2}}{2}$, we have

$$
\rho_{t} \frac{\left|(\log \rho)_{r}\right|^{2} r^{2}}{2}+\frac{\left|(\log \rho)_{r}\right|^{2}}{2}\left(\rho u r^{2}\right)_{r}=0 .
$$


Furthermore, $(3.5)_{1}$ implies that

$$
\begin{aligned}
& \rho\left(\frac{\left|(\log \rho)_{r}\right|^{2} r^{2}}{2}\right)_{t}+\left(\frac{\left|(\log \rho)_{r}\right|^{2}}{2}\right)_{r} \rho u r^{2} \\
& \quad=-\rho_{r} u_{r r} r^{2}-\rho_{r} u_{r}(\log \rho)_{r} r^{2}-2 \rho_{r} u_{r} r+2 \rho_{r} u .
\end{aligned}
$$

Adding (3.18) and (3.19), then integrating the result equation over $(0, R)$, one gets

$$
\frac{d}{d t} \int_{0}^{R} \frac{\rho\left|(\log \rho)_{r}\right|^{2}}{2} r^{2} d r=\int_{0}^{R}\left(2 \rho_{r} u-\rho_{r} u_{r r} r^{2}-\rho_{r} u_{r}(\log \rho)_{r} r^{2}-2 \rho_{r} u_{r} r\right) d r
$$

Using integration by parts and $(3.5)_{1}$, we also get that

$$
\begin{aligned}
& \frac{d}{d t} \int_{0}^{R}(\log \rho)_{r} \rho u r^{2} d r \\
& =\int_{0}^{R}(\log \rho)_{r} \partial_{t}(\rho u) r^{2} d r+\int_{0}^{R}\left(\rho u r^{2}\right)_{r}(\log \rho)_{r} u d r \\
& \quad+\int_{0}^{R}\left(\rho_{r} u u_{r} r^{2}+\rho u_{r}^{2} r^{2}+4 \rho u u_{r} r+2 \rho_{r} u^{2} r+4 \rho u^{2}\right) d r
\end{aligned}
$$

It follows from $(3.5)_{2}$ that

$$
\begin{aligned}
\int_{0}^{R}(\log \rho)_{r} \partial_{t}(\rho u) r^{2} d r \\
\quad=\int_{0}^{R} \rho \phi_{r}(\log \rho)_{r} r^{2} d r-\int_{0}^{R} \frac{4}{\gamma}\left[\left(\rho^{\frac{\gamma}{2}}\right)_{r} r\right]^{2} d r-\int_{0}^{R}\left(\rho u r^{2}\right)_{r}(\log \rho)_{r} u d r \\
\quad-\int_{0}^{R} \rho_{r} u u_{r} r^{2} d r+\int_{0}^{R}\left(\rho_{r} u_{r r} r^{2}+\rho_{r} u_{r}(\log \rho)_{r} r^{2}+2 \rho_{r} u_{r} r-2 \rho_{r} u\right) d r .
\end{aligned}
$$

Returning to (3.21), we have

$$
\begin{aligned}
& \frac{d}{d t} \int_{0}^{R}(\log \rho)_{r} \rho u r^{2} d r \\
& =\int_{0}^{R} \rho \phi_{r}(\log \rho)_{r} r^{2} d r-\int_{0}^{R} \frac{4}{\gamma}\left(\left(\rho^{\frac{\gamma}{2}}\right)_{r} r\right)^{2} d r+\int_{0}^{R}\left(\rho_{r} u_{r r} r^{2}+\rho_{r} u_{r}(\log \rho)_{r} r^{2}\right. \\
& \left.\quad+2 \rho_{r} u_{r} r-2 \rho_{r} u+\rho u_{r}^{2} r^{2}+4 \rho u u_{r} r+2 \rho_{r} u^{2} r+4 \rho u^{2}\right) d r
\end{aligned}
$$

Summing (3.20) and (3.23) then using integration by parts, we have

$$
\begin{aligned}
& \frac{d}{d t} \int_{0}^{R}\left\{(\log \rho)_{r} \rho u+\frac{\rho\left|(\log \rho)_{r}\right|^{2}}{2}\right\} r^{2} d r+\int_{0}^{R} \frac{4}{\gamma}\left[\left(\rho^{\frac{\gamma}{2}}\right)_{r} r\right]^{2} d r \\
& \quad=\int_{0}^{R} \rho \phi_{r}(\log \rho)_{r} r^{2} d r+\int_{0}^{R}\left(\rho u_{r}^{2} r^{2}+4 \rho u u_{r} r+2 \rho_{r} u^{2} r+4 \rho u^{2}\right) d r \\
& \quad=\int_{0}^{R} \rho \phi_{r}(\log \rho)_{r} r^{2} d r+\int_{0}^{R} \rho\left(u_{r}^{2} r^{2}+2 u^{2}\right) d r
\end{aligned}
$$


Combining (3.24) with inequality (3.14), one obtains

$$
\begin{aligned}
& \frac{d}{d t} \int_{0}^{R}\left\{\frac{1}{2} \rho\left|u+(\log \rho)_{r}\right|^{2}+\frac{1}{2} \phi_{r}^{2}+\frac{\rho^{\gamma}}{\gamma-1}\right\} r^{2} d r+\int_{0}^{R} \frac{4}{\gamma}\left(\left(\rho^{\frac{\gamma}{2}}\right)_{r} r\right)^{2} d r \\
& \quad \leq \int_{0}^{R} \rho \phi_{r}(\log \rho)_{r} r^{2} d r .
\end{aligned}
$$

We now estimate the right-hand side of (3.25). Integrating by parts and using the boundary condition (3.3), as well as the property of function $b(x)$, we obtain the following:

$$
\begin{aligned}
\int_{0}^{R} \rho \phi_{r}(\log \rho)_{r} r^{2} d r & =\int_{\Omega} \nabla \Phi \cdot \nabla \rho d x \\
& =\int_{\Omega} \nabla \Phi \cdot \nabla(\rho-b) d x+\int_{\Omega} \nabla \Phi \cdot \nabla b d x \\
& =-\int_{\Omega}(\rho-b)^{2} d x-\int_{\Omega} \rho b d x+\int_{\Omega} b^{2} d x \\
& \leq-\int_{\Omega}(\rho-b)^{2} d x-\int_{\Omega} \rho b d x+C \\
& =-\int_{0}^{R}\left\{(\rho-b)^{2}+\rho b\right\} r^{2} d r+C .
\end{aligned}
$$

Substituting the above inequality into (3.25), one obtains the desired estimate (3.16), and so the lemma is proved.

Now we give a definition of weak solutions to (3.1) with the initial and boundary conditions (3.2) and (3.3).

Definition 3.3 A pair $(\rho, U)$ with $\rho \geq 0$ a.e. is said to be a weak solution to (3.1) provided that $\rho \in L^{\infty}\left(0, T ; L^{1}(\Omega) \cap L^{\gamma}(\Omega)\right) \cap C\left([0, \infty) ; W^{1, \infty}(\Omega)^{*}\right), \sqrt{\rho} \in L^{\infty}\left(0, T ; H^{1}(\Omega)\right), \sqrt{\rho} U \in$ $L^{\infty}\left(0, T ; L^{2}(\Omega)\right)$, where $W^{1, \infty}(\Omega)^{*}$ is the dual space of $W^{1, \infty}(\Omega)$, and the equations are satisfied in sense of distributions. That is, it holds for any $t_{2}>t_{1} \geq 0$ and $\psi \in C^{1}(\bar{\Omega} \times[0, T])$ that

$$
\left.\int_{\Omega} \rho \psi d x\right|_{t_{1}} ^{t_{2}}=\int_{t_{1}}^{t_{2}} \int_{\Omega}\left(\rho \psi_{t}+\rho U \cdot \nabla \psi\right) d x d t
$$

and for $\psi=\left(\psi^{1}, \psi^{2}, \psi^{3}\right) \in C^{1}(\bar{\Omega} \times[0, T])$ satisfying $\psi(\mathbf{x}, t)=0$ on $\partial \Omega$ and $\psi(\mathbf{x}, T)=0$, it holds that

$$
\begin{aligned}
& \int_{\Omega} \mathbf{m}_{0} \cdot \psi(\cdot, 0) d x+\int_{0}^{T} \int_{\Omega}\left\{\sqrt{\rho}(\sqrt{\rho} U) \cdot \partial_{t} \psi+\sqrt{\rho} U \otimes \sqrt{\rho} U: \nabla \psi\right\} d x d t \\
& +\int_{0}^{T} \int_{\Omega} \rho^{\gamma} \operatorname{div} \psi d x d t-\langle\rho \nabla U, \nabla \psi\rangle=\int_{0}^{T} \int_{\Omega} \rho \nabla \Phi \psi d x d t
\end{aligned}
$$

where $\mathbf{m}_{0}=m_{0} \frac{\mathbf{x}}{r}$ and the diffusion term is defined for any $\psi \in C^{1}(\bar{\Omega} \times[0, T])$ as

$$
\begin{aligned}
\langle\rho \nabla U, \nabla \psi\rangle= & -\int_{0}^{T} \int_{\Omega} \sqrt{\rho}(\sqrt{\rho} U) \cdot \Delta \psi d x d t \\
& -2 \int_{0}^{T} \int_{\Omega}(\sqrt{\rho} U) \cdot(\nabla \sqrt{\rho} \cdot \nabla) \psi d x d t
\end{aligned}
$$


and

$$
\Delta \Phi=\rho-b \quad \text { a.e. }
$$

In this paper, the initial data are assumed to satisfy

$$
\begin{aligned}
& \rho_{0} \geq 0 \quad \text { a.e. in } \Omega ; \quad \mathbf{m}_{0}=0 \quad \text { a.e. on }\left\{x \in \Omega \mid \rho_{0}(x)=0\right\} ; \\
& \rho_{0} \in W^{1,2}(\Omega) ; \quad \frac{\mathbf{m}_{0}^{2}}{\rho_{0}} \in L^{1}(\Omega) ; \quad \frac{\mathbf{m}_{0}^{2+\eta}}{\rho_{0}^{1+\eta}} \in L^{1}(\Omega)
\end{aligned}
$$

with $\eta \in(0,1)$ a suitably small constant. It follows from the above assumption that

$$
\rho_{0} U_{0}^{2+\eta} \in L^{1}(\Omega) ; \quad \rho_{0} U_{0}^{2} \in L^{1}(\Omega)
$$

Then we have the following global existence result.

Theorem 3.4 Let $N=3,1<\gamma<3$. Assume that (3.31) holds and the initial data have the form

$$
\rho_{0}=\rho_{0}(|\mathbf{x}|), \quad U_{0}=u_{0}(|\mathbf{x}|) \frac{\mathbf{x}}{r}
$$

then the initial boundary value problem (3.1)-(3.3) has a global spherically symmetric weak solution

$$
\rho=\rho(|\mathbf{x}|, t), \quad U=u(|\mathbf{x}|, t) \frac{\mathbf{x}}{r}
$$

in the sense of Definition 3.3 satisfying for all $T>0$,

$$
\begin{aligned}
& \rho(\mathbf{x}, t) \in C\left([0, T] ; L^{\frac{3}{2}}(\Omega)\right), \quad \sqrt{\rho} U \in L^{\infty}\left(0, T ; L^{2}(\Omega)\right), \\
& \int_{\Omega} \rho(\mathbf{x}, t) d x=\int_{\Omega} \rho_{0}(\mathbf{x}) d x, \\
& \sup _{t \in[0, T]} \int_{\Omega}\left(\rho^{\gamma}+|\sqrt{\rho} U|^{2}\right) d x+\int_{0}^{T} \int_{\Omega}|\sqrt{\rho} \nabla U|^{2} d x d t \leq C,
\end{aligned}
$$

and

$$
\sup _{t \in[0, T]} \int_{\Omega}|\nabla \sqrt{\rho}|^{2} d x+\int_{0}^{T} \int_{\Omega}\left|\sqrt{\rho^{\frac{\gamma}{2}}}\right|^{2} d x d t \leq C,
$$

where $C$ is a constant.

Remark 3.5 It can be checked easily that, for $N=2$, the conclusions in Theorem 3.4 hold true for any $\gamma>1$.

\section{Approximate solutions}

The crucial step in the proof of Theorem 3.4 is to construct smooth approximate solutions satisfying the a priori estimates required in the $L^{1}$-stability analysis. The key point is to obtain lower and upper bounds of the density. In this section, we construct the suitable approximate solutions and obtain their estimates. 


\subsection{Approximate system in Euler coordinates and the estimates}

To this end, we study the following system as an approximate system of (3.1):

$$
\left\{\begin{array}{l}
\rho_{t}+\operatorname{div}(\rho U)=0, \\
(\rho U)_{t}+\operatorname{div}(\rho U \otimes U)-\operatorname{div}\left(\left(\rho+\epsilon \rho^{\frac{3}{4}}\right) \nabla U\right)+\nabla\left(\frac{\epsilon}{4} \rho^{\frac{3}{4}} \operatorname{div} U\right)+\nabla \rho^{\gamma}=\rho \nabla \Phi, \\
\Delta \Phi=\rho-b,
\end{array}\right.
$$

where $\epsilon>0$ is a constant. Considering a spherically symmetric solution, system (4.1) becomes

$$
\left\{\begin{array}{l}
\rho_{t}+(\rho u)_{r}+\frac{2 \rho u}{r}=0, \\
(\rho u)_{t}+\left(\rho u^{2}+\rho^{\gamma}\right)_{r}+\frac{2 \rho u^{2}}{r}+\left(\rho+\epsilon \rho^{\frac{3}{4}}\right)_{r} \frac{2 u}{r}=\left(\left(\rho+\frac{3}{4} \epsilon \rho^{\frac{3}{4}}\right)\left(u_{r}+\frac{2 u}{r}\right)\right)_{r}+\rho \phi_{r}, \\
\phi_{r r}+\frac{2}{r} \phi_{r}=\rho-b,
\end{array}\right.
$$

for $0<\epsilon<r<R$ with the following initial condition:

$$
(\rho, \rho u, \phi)(r, 0)=\left(\rho_{0}+\epsilon, m_{0}, \phi_{0}\right)
$$

and the boundary conditions

$$
\left.u\right|_{r=\epsilon}=\left.u\right|_{r=R}=0,\left.\quad \phi_{r}\right|_{r=\epsilon}=\left.\phi_{r}\right|_{r=R}=0 .
$$

For the approximate solutions which will possess a lower bound of the density, the boundary conditions (4.4) are equivalent to $\left.\left(\rho u, \phi_{r}\right)(r, t)\right|_{r=\epsilon}=0,\left.\left(\rho u, \phi_{r}\right)(r, t)\right|_{r=R}=0$. Without loss of generality, it is assumed in this section that the initial data are smooth enough and satisfy the bounds in (3.31) with constants independent of $\epsilon$.

In the following, we will state the energy and entropy estimates which have been proved in the preceding section for these approximate solutions.

Lemma 4.1 Let $\left(\rho^{\epsilon}, u^{\epsilon}\right)$ be smooth solutions of (4.2)-(4.4) such that $\rho^{\epsilon}>0$. Then there exists a constant $C$ independent of $\epsilon$ such that

$$
\begin{aligned}
& \int_{\epsilon}^{R} \rho^{\epsilon}(r, t) r^{2} d r \leq C, \\
& \int_{\epsilon}^{R}\left\{\frac{1}{2} \rho^{\epsilon}\left(u^{\epsilon}\right)^{2}+\frac{1}{\gamma-1}\left(\rho^{\epsilon}\right)^{\gamma}+\frac{1}{2}\left(\phi_{r}^{\epsilon}\right)^{2}\right\} r^{2} d r \\
& \quad+\int_{0}^{T} \int_{\epsilon}^{R}\left\{\rho^{\epsilon}+\frac{\epsilon}{4}\left(\rho^{\epsilon}\right)^{\frac{3}{4}}\right\}\left\{\left(u_{r}^{\epsilon}\right)^{2} r^{2}+2\left(u^{\epsilon}\right)^{2}\right\} d r d t \leq C, \\
& \int_{\epsilon}^{R}\left\{\frac{1}{2} \rho^{\epsilon}\left|u^{\epsilon}+\left(\log \rho^{\epsilon}\right)_{r}+\frac{3}{4} \epsilon\left(\rho^{\epsilon}\right)^{-\frac{5}{4}} \rho_{r}^{\epsilon}\right|^{2}+\frac{1}{2}\left(\phi_{r}^{\epsilon}\right)^{2}+\frac{1}{\gamma-1}\left(\rho^{\epsilon}\right)^{\gamma}\right\} r^{2} d r \\
& \quad+\int_{0}^{T} \int_{\epsilon}^{R}\left\{\gamma\left(\rho^{\epsilon}\right)^{\gamma-2}+\frac{3}{4} \epsilon \gamma\left(\rho^{\epsilon}\right)^{\gamma-\frac{9}{4}}\right\}\left|\rho_{r}^{\epsilon}\right|^{2} r^{2} d r d t \\
& \quad+\int_{0}^{T} \int_{\epsilon}^{R}\left\{\left(\rho^{\epsilon}-b\right)^{2}+b \rho^{\epsilon}\right\} r^{2} d r d t \leq C .
\end{aligned}
$$


To simplify the presentation, we drop the superscript $\epsilon$ in the rest of this section.

Lemma 4.2 Given $\epsilon>0$, there is an absolute constant $C$, which is independent of $\epsilon$, such that

$$
0 \leq \rho(r, t) \leq \frac{C}{\epsilon^{2}},
$$

for $\epsilon \leq r \leq R$ and $t \geq 0$.

Proof Let $r(t)$ denote a particle path satisfying

$$
\frac{d r(t)}{d t}=u(r(t), t)
$$

Then along the particle path, $(4.2)_{1}$ can be solved to get

$$
\rho(r(t), t) r^{2}(t)=\left(\rho_{0}(r(0))+\epsilon\right) r^{2}(0) e^{-\int_{0}^{t} u_{r}(r(s), s) d s},
$$

which implies that $\rho>0$, provided $\rho_{0} \geq 0$.

Moreover,

$$
\begin{aligned}
& \int_{\epsilon}^{R} \frac{1}{2} \rho\left\{(\log \rho)_{r}^{2}+\frac{3}{2} \epsilon \rho^{-\frac{5}{4}} \rho_{r}^{2}+\frac{9}{16} \epsilon^{2} \rho^{-\frac{3}{2}} \rho_{r}^{2}\right\} r^{2} d r \\
& \quad=\int_{\epsilon}^{R} \frac{1}{2} \rho\left|(\log \rho)_{r}+\frac{3}{4} \epsilon \rho^{-\frac{5}{4}} \rho_{r}\right|^{2} r^{2} d r \\
& \quad \leq \int_{\epsilon}^{R} \frac{1}{2} \rho\left|(\log \rho)_{r}+\frac{3}{4} \epsilon \rho^{-\frac{5}{4}} \rho_{r}+u\right|^{2} r^{2} d r+\int_{\epsilon}^{R} \frac{1}{2} \rho u_{r} r^{2} d r \\
& \quad \leq C
\end{aligned}
$$

due to (4.6) and (4.7), then we obtain

$$
\int_{\epsilon}^{R} \frac{\rho_{r}^{2}}{\rho} r^{2} d r \leq C
$$

for some absolute constant $C$ independent of $\epsilon$. Then, it follows from (4.5) and (4.12) that, for $\epsilon \leq r \leq R$,

$$
\begin{aligned}
\rho(r, t) & \leq \int_{\epsilon}^{R} \rho(r, t) d r+\int_{\epsilon}^{R}\left|\rho_{r}(r, t)\right| d r \\
& \leq \frac{1}{\epsilon^{2}} \int_{\epsilon}^{R} \rho(r, t) r^{2} d r+\frac{1}{\epsilon^{2}} \int_{\epsilon}^{R} \sqrt{\rho} \frac{\left|\rho_{r}(r, t)\right|}{\sqrt{\rho}} r^{2} d r \\
& \leq \frac{C}{\epsilon^{2}}
\end{aligned}
$$

for all $t \geq 0$. The lemma is proved. 


\subsection{Approximate system in Lagrangian coordinates and the estimates}

To obtain the a priori estimates for the velocity of the approximate solutions, the key point is to derive lower bounds of the density. To this end, we introduce Lagrangian coordinates for the radial system (4.2) as follows. Let $\epsilon>0$ be fixed and define

$$
x(r, t)=\int_{\epsilon}^{r} \rho r^{2} d r, \quad \tau=t .
$$

Set $\int_{\epsilon}^{1} \rho r^{2} d r=1$ for $\epsilon>0$, without loss of generality. Then,

$$
\frac{\partial x}{\partial r}=\rho r^{2}, \quad \frac{\partial x}{\partial t}=-\rho u r^{2}, \quad \frac{\partial \tau}{\partial r}=0, \quad \frac{\partial \tau}{\partial t}=1 .
$$

Then system (4.2) becomes

$$
\left\{\begin{array}{l}
\rho_{\tau}+\rho^{2}\left(r^{2} u\right)_{x}=0, \\
r^{-2} u_{\tau}+\left(\rho^{\gamma}\right)_{x}=\left\{\left(\rho^{2}+\frac{3}{4} \epsilon \rho^{\frac{7}{4}}\right)\left(r^{2} u\right)_{x}\right\}_{x}-\left(\rho+\epsilon \rho^{\frac{3}{4}}\right)_{x} \frac{2 u}{r}+\frac{x}{r^{4}}-\frac{g_{b}}{r^{2}}
\end{array}\right.
$$

for $\tau>0$ and $0 \leq x \leq 1$, where $g_{b}(x, \tau):=f_{b}(r(x, \tau))=\frac{1}{r^{2}(x, \tau)} \int_{\epsilon}^{r(x, \tau)} b(s) s^{2} d s$. The corresponding initial data and the boundary conditions are

$$
\begin{aligned}
& (\rho, \rho u, \phi)(\cdot, 0)=\left(\rho_{0}+\epsilon, m_{0}, \phi_{0}\right), \\
& u(0, \tau)=0, \quad u(1, \tau)=0 .
\end{aligned}
$$

Lemma 4.3 For all $\tau \in[0, T]$, it holds that

$$
\begin{aligned}
& \int_{0}^{1}\left(\frac{u^{2}}{2}+\frac{\rho^{\gamma-1}}{\gamma-1}+\frac{x}{r}+r\right) d x+\int_{0}^{\tau} \int_{0}^{1}\left(\frac{2 u^{2}}{r^{2}}+\rho^{2} u_{x}^{2} r^{4}\right) d x d s+\int_{0}^{\tau} \int_{0}^{1} \epsilon \frac{u^{2}}{\rho^{\frac{1}{4}} r^{2}} d x d s \\
& \quad+\int_{0}^{\tau} \int_{0}^{1} \epsilon \rho^{\frac{7}{4}} u_{x}^{2} r^{4} d x d s \leq \int_{0}^{1}\left(\frac{u_{0}^{2}}{2}+\frac{\rho_{0}^{\gamma-1}}{\gamma-1}+\frac{x}{r_{0}}+r_{0}\right) d x \\
& 0 \leq \rho(x, \tau) \leq C(\epsilon, T), \\
& \epsilon \leq r(x, \tau) \leq R \\
& \int_{0}^{1} u^{4} d x+\int_{0}^{\tau} \int_{0}^{1}\left(\frac{4 u^{4}}{r^{2}}+6 \rho^{2} u^{2} u_{x}^{2} r^{4}+\frac{2 \epsilon u^{4}}{\rho^{\frac{1}{4}} r^{2}}+\epsilon \rho^{\frac{7}{4}} u^{2} u_{x}^{2} r^{4}\right) d x d s \\
& \quad \leq \int_{0}^{1} u_{0}^{4} d x+C(\epsilon, T) .
\end{aligned}
$$

Proof Multiplying $(4.16)_{2}$ by $u r^{2}$, a direct computation gives

$$
\begin{aligned}
& \frac{d}{d \tau} \int_{0}^{1}\left(\frac{u^{2}}{2}+\frac{\rho^{\gamma-1}}{\gamma-1}+\frac{x}{r}+g_{b} r\right) d x+\int_{0}^{1}\left(\rho^{2}+\frac{3}{4} \epsilon \rho^{\frac{7}{4}}\right)\left(4 \frac{u^{2}}{\rho^{2} r^{2}}+4 \frac{u u_{x} r}{\rho}+u_{x}^{2} r^{4}\right) d x \\
& \quad=4 \int_{0}^{1}\left(\rho+\epsilon \rho^{\frac{3}{4}}\right) u u_{x} r d x+2 \int_{0}^{1}\left(1+\frac{\epsilon}{\rho^{\frac{1}{4}}}\right) \frac{u^{2}}{r^{2}} d x .
\end{aligned}
$$


Using Young inequality, we obtain

$$
\begin{aligned}
& \frac{d}{d \tau} \int_{0}^{1}\left(\frac{u^{2}}{2}+\frac{\rho^{\gamma-1}}{\gamma-1}+\frac{x}{r}+g_{b} r\right) d x+\int_{0}^{1}\left(\frac{2 u^{2}}{r^{2}}+\rho^{2} u_{x}^{2} r^{4}\right) d x+\left(1-\frac{\lambda}{2}\right) \int_{0}^{1} \frac{\epsilon u^{2}}{\rho^{\frac{1}{4}} r^{2}} d x \\
& \quad+\left(\frac{3}{4}-\frac{1}{2 \lambda}\right) \int_{0}^{1} \epsilon \rho^{\frac{7}{4}} u_{x}^{2} r^{4} d x \leq 0, \quad \forall \lambda \in\left(\frac{2}{3}, 2\right) .
\end{aligned}
$$

Since $b$ is a smooth function and satisfies (1.3), there exist constants $C_{1}, C_{2}>0$ such that

$$
C_{1} \leq g_{b}(x, \tau):=f_{b}(r(x, \tau))=\frac{1}{r^{2}(x, \tau)} \int_{\epsilon}^{r(x, \tau)} b(s) s^{2} d s \leq C_{2}
$$

Integrating both sides of (4.24) with respect to $\tau$ and using (4.25), we can get

$$
\begin{aligned}
& \int_{0}^{1}\left(\frac{u^{2}}{2}+\frac{\rho^{\gamma-1}}{\gamma-1}+\frac{x}{r}+r\right) d x+\int_{0}^{\tau} \int_{0}^{1}\left(\frac{2 u^{2}}{r^{2}}+\rho^{2} u_{x}^{2} r^{4}\right) d x d s \\
& +\left(1-\frac{\lambda}{2}\right) \int_{0}^{\tau} \int_{0}^{1} \frac{\epsilon u^{2}}{\rho^{\frac{1}{4}} r^{2}} d x d s+\left(\frac{3}{4}-\frac{1}{2 \lambda}\right) \int_{0}^{\tau} \int_{0}^{1} \epsilon \rho^{\frac{7}{4}} u_{x}^{2} r^{4} d x d s \\
& \leq \int_{0}^{1}\left(\frac{u_{0}^{2}}{2}+\frac{\left(\rho_{0}+\epsilon\right)^{\gamma-1}}{\gamma-1}+\frac{x}{r_{0}}+r_{0}\right) d x, \quad r_{0}:=r(x, \tau=0), \lambda \in\left(\frac{2}{3}, 2\right)
\end{aligned}
$$

thus (4.19) holds.

Multiplying $(4.16)_{2}$ by $u^{3} r^{2}$, we deduce

$$
\begin{gathered}
\frac{1}{4} \frac{d}{d \tau} \int_{0}^{1} u^{4} d x+\int_{0}^{1}\left(\rho^{2}+\frac{3}{4} \epsilon \rho^{\frac{7}{4}}\right)\left(r^{2} u\right)_{x}^{2} u^{2} d x+2 \int_{0}^{1}\left(\rho^{2}+\frac{3}{4} \epsilon \rho^{\frac{7}{4}}\right)\left(r^{2} u\right)_{x} r^{2} u^{2} u_{x} d x \\
\quad=\int_{0}^{1} \rho^{\gamma}\left(u^{3} r^{2}\right)_{x} d x+\int_{0}^{1}\left(\rho+\epsilon \rho^{\frac{3}{4}}\right)\left(2 r u^{4}\right)_{x} d x+\int_{0}^{1}\left(\frac{x}{r^{2}}-g_{b}\right) u^{3} d x
\end{gathered}
$$

i.e.,

$$
\begin{aligned}
\frac{1}{4} & \frac{d}{d \tau} \int_{0}^{1} u^{4} d x+\int_{0}^{1}\left(3 \rho^{2} u^{2} u_{x}^{2} r^{4}+\frac{2 u^{4}}{r^{2}}\right) d x+\int_{0}^{1}\left(\frac{9}{4} \epsilon \rho^{\frac{7}{4}} u^{2} u_{x}^{2} r^{4}+\frac{\epsilon u^{4}}{\rho^{\frac{1}{4}} r^{2}}\right) d x \\
= & \int_{0}^{1} 2 \epsilon \rho^{\frac{3}{4}} u^{3} u_{x} r d x+\int_{0}^{1}\left(\rho^{\gamma-1} \frac{2 u^{3}}{r}+3 \rho^{\gamma} u^{2} u_{x} r^{2}\right) d x \\
& +\int_{0}^{1}\left(\frac{x}{r^{2}}-g_{b}\right) u^{3} d x
\end{aligned}
$$

Using Hölder and Young inequalities together with Lemma 3.2, we can estimate the right-hand side of (4.28) as follows:

$$
\begin{aligned}
\int_{0}^{1} 2 \epsilon \rho^{\frac{3}{4}} u^{3} u_{x} r d x & \leq \frac{1}{2} \int_{0}^{1} \frac{\epsilon u^{4}}{\rho^{\frac{1}{4}} r^{2}} d x+2 \int_{0}^{1} \epsilon \rho^{\frac{7}{4}} u^{2} u_{x}^{2} r^{4} d x \\
\int_{0}^{1} \rho^{\gamma-1} \frac{2 u^{3}}{r} d x & \leq \frac{1}{2} \int_{0}^{1} \frac{u^{4}}{r^{2}} d x+C \int_{0}^{1} \rho^{4(\gamma-1)} r^{2} d x \\
& \leq \frac{1}{2} \int_{0}^{1} \frac{u^{4}}{r^{2}} d x+C(\epsilon)
\end{aligned}
$$


Gui and Song Boundary Value Problems

(2019) 2019:157

Page 14 of 40

$$
\begin{aligned}
\int_{0}^{1} 3 \rho^{\gamma} u^{2} u_{x} r^{2} d x & \leq \frac{3}{2} \int_{0}^{1} \rho^{2(\gamma-1)} u^{2} d x+\frac{3}{2} \int_{0}^{1} \rho^{2} u^{2} u_{x}^{2} r^{4} d x \\
& \leq \frac{3}{2}\left(\int_{0}^{1} \rho^{4(\gamma-1)} r^{2} d x\right)^{\frac{1}{2}}\left(\int_{0}^{1} \frac{u^{4}}{r^{2}} d x\right)^{\frac{1}{2}}+\frac{3}{2} \int_{0}^{1} \rho^{2} u^{2} u_{x}^{2} r^{4} d x \\
& \leq \frac{1}{2} \int_{0}^{1} \frac{u^{4}}{r^{2}} d x+\frac{3}{2} \int_{0}^{1} \rho^{2} u^{2} u_{x}^{2} r^{4} d x+C(\epsilon), \\
\int_{0}^{1}\left(\frac{x}{r^{2}}-g_{b}\right) u^{3} d x & \leq C \int_{0}^{1}|u|^{3} d x \leq C \int_{0}^{1} u^{4} d x .
\end{aligned}
$$

Putting the above estimates (4.29) - (4.32) into (4.28), we easily obtain

$$
\begin{aligned}
& \frac{d}{d \tau} \int_{0}^{1} u^{4} d x+\int_{0}^{1}\left(\frac{4 u^{4}}{r^{2}}+6 \rho^{2} u^{2} u_{x}^{2} r^{4}+\frac{2 \epsilon u^{4}}{\rho^{\frac{1}{4}} r^{2}}+\epsilon \rho^{\frac{7}{4}} u^{2} u_{x}^{2} r^{4}\right) d x \\
& \quad \leq C(\epsilon)+C \int_{0}^{1} u^{4} d x
\end{aligned}
$$

By applying Gronwall's inequality to (4.33), we have

$$
\int_{0}^{1} u^{4} d x \leq \exp \left\{\int_{0}^{\tau} C d s\left(\int_{0}^{\tau} u_{0}^{4} d s+\int_{0}^{\tau} C d s\right)\right\} \leq C(T)+C \int_{0}^{1} u_{0}^{4} d x .
$$

Thus, it is easy to obtain (4.22). The proof of this lemma is completed.

Lemma 4.4 There is a positive constant $C=C\left(\epsilon, T,\left\|u_{0}\right\|_{L^{4}},\left\|\left(\rho_{0}^{\frac{3}{4}}\right)_{x}\right\|_{L^{4}}\right)$ such that

$$
\int_{0}^{1}\left(\left(\rho^{\frac{3}{4}}\right)_{x}\right)^{4}(x, \tau) d x \leq C, \quad \forall \tau \in[0, T]
$$

Proof By making use of (4.16), we have

$$
\left(\rho+\epsilon \rho^{\frac{3}{4}}\right)_{x \tau}=-\left\{\left(\rho^{2}+\frac{3}{4} \epsilon \rho^{\frac{7}{4}}\right)\left(r^{2} u\right)_{x}\right\}_{x},
$$

then we can rewrite $(4.16)_{2}$ as follows:

$$
r^{2}\left(\rho+\epsilon \rho^{\frac{3}{4}}\right)_{x \tau}+\left(\rho+\epsilon \rho^{\frac{3}{4}}\right)_{x} 2 u r=-u_{\tau}-\left(\rho^{\gamma}\right)_{x} r^{2}+\frac{x}{r^{2}}-g_{b},
$$

i.e.,

$$
\left\{r^{2}\left(\rho+\epsilon \rho^{\frac{3}{4}}\right)_{x}\right\}_{\tau}=-u_{\tau}-\left(\rho^{\gamma}\right)_{x} r^{2}+\frac{x}{r^{2}}-g_{b},
$$

where we have used the fact that $\frac{\partial r}{\partial \tau}=u$.

Integrating (4.38) over $[0, t]$ shows

$$
\begin{aligned}
& -u(x, t)+u_{0}(x)-\int_{0}^{t}\left(\rho^{\gamma}\right)_{x} r^{2}(x, s) d s+\int_{0}^{t}\left(\frac{x}{r^{2}}-g_{b}\right) d s \\
& =r^{2}\left(\rho+\epsilon \rho^{\frac{3}{4}}\right)_{x}-r_{0}^{2}\left\{\rho_{0}+\epsilon+\epsilon\left(\rho_{0}+\epsilon\right)^{\frac{3}{4}}\right\}_{x} \\
& =r^{2}\left(\frac{4}{3} \rho^{\frac{1}{4}}+\epsilon\right) \partial_{x}\left(\rho^{\frac{3}{4}}\right)-r_{0}^{2}\left\{\frac{4}{3} \rho_{0}^{\frac{1}{4}}+\epsilon \rho_{0}^{\frac{1}{4}}\left(\rho_{0}+\epsilon\right)^{-\frac{1}{4}}\right\} \partial_{x}\left(\rho_{0}^{\frac{3}{4}}\right),
\end{aligned}
$$


Gui and Song Boundary Value Problems

(2019) 2019:157

Page 15 of 40

i.e.,

$$
\begin{aligned}
r^{2}( & \left.\frac{4}{3} \rho^{\frac{1}{4}}+\epsilon\right) \partial_{x}\left(\rho^{\frac{3}{4}}\right) \\
= & -u(x, t)+u_{0}(x)-\int_{0}^{t}\left(\rho^{\gamma}\right)_{x} r^{2}(x, t) d s \\
& \quad+\int_{0}^{t}\left(\frac{x}{r^{2}}-g_{\triangle}\right) d s+r_{0}^{2}\left\{\frac{4}{3} \rho_{0}^{\frac{1}{4}}+\epsilon \rho_{0}^{\frac{1}{4}}\left(\rho_{0}+\epsilon\right)^{-\frac{1}{4}}\right\} \partial_{x}\left(\rho_{0}^{\frac{3}{4}}\right) .
\end{aligned}
$$

Multiplying (4.40) by $\left\{\partial_{x}\left(\rho^{\frac{3}{4}}\right) r^{2}\right\}^{3}$ and integrating over $[0,1]$ with respect to $x$, one gets

$$
\begin{aligned}
\int_{0}^{1} & \left(\frac{4}{3} \rho^{\frac{1}{4}}+\epsilon\right)\left\{\partial_{x}\left(\rho^{\frac{3}{4}}\right) r^{2}\right\}^{4} d x \\
= & \int_{0}^{1}\left\{-u(x, t)+u_{0}(x)-\int_{0}^{t}\left(\rho^{\gamma}\right)_{x} r^{2}(x, t) d s\right. \\
& \left.\left.+\int_{0}^{t}\left(\frac{x}{r^{2}}-g_{\Delta}\right) d s+r_{0}^{2}\left\{\frac{4}{3} \rho_{0}^{\frac{1}{4}}+\epsilon \rho_{0}^{\frac{1}{4}} \rho_{0}+\epsilon\right)^{-\frac{1}{4}}\right\} \partial_{x}\left(\rho_{0}^{\frac{3}{4}}\right)\right\}
\end{aligned}
$$

and

$$
\begin{aligned}
& \left\{\partial_{x}\left(\rho^{\frac{3}{4}}\right) r^{2}\right\}^{3} d x \\
& \leq\left\{\int_{0}^{1}\left(\partial_{x}\left(\rho^{\frac{3}{4}}\right) r^{2}\right)^{4} d x\right\}^{\frac{3}{4}}\left\{\left\|-u(x, t)+u_{0}(x)\right\|_{L^{4}}+\left\|\int_{0}^{t}\left(\rho^{\gamma}\right)_{x} r^{2}(x, t) d s\right\|_{L^{4}}\right. \\
& \left.\quad+\left\|\int_{0}^{t}\left(\frac{x}{r^{2}}-g_{\Delta}\right) d s\right\|_{L^{4}}+\left\|r_{0}^{2}\left\{\frac{4}{3} \rho_{0}^{\frac{1}{4}}+\epsilon \rho_{0}^{\frac{1}{4}}\left(\rho_{0}+\epsilon\right)^{-\frac{1}{4}}\right\} \partial_{x}\left(\rho_{0}^{\frac{3}{4}}\right)\right\|_{L^{4}}\right\} \\
& \leq C\left\{\int_{0}^{1}\left(\partial_{x}\left(\rho^{\frac{3}{4}}\right) r^{2}\right)^{4} d x\right\}^{\frac{3}{4}}\left\{C\left(\epsilon, T,\left\|u_{0}\right\|_{L^{4}},\left\|\partial_{x}\left(\rho_{0}^{\frac{3}{4}}\right)\right\|_{L^{4}}\right)\right. \\
& \left.\quad+\left\|\int_{0}^{t}\left(\rho^{\gamma}\right)_{x^{2}} r^{2}(x, t) d s\right\|_{L^{4}}\right\} .
\end{aligned}
$$

Using Lemma 4.3 and Young's inequality, one gets from (4.41) that

$$
\begin{aligned}
\epsilon \int_{0}^{1} & \left\{\partial_{x}\left(\rho^{\frac{3}{4}}\right) r^{2}\right\}^{4} d x \\
\leq & \frac{\epsilon}{2} \int_{0}^{1}\left\{\partial_{x}\left(\rho^{\frac{3}{4}}\right) r^{2}\right\}^{4} d x+C \int_{0}^{t} \int_{0}^{1}\left(\partial_{x} \rho^{\gamma}\right)^{4} d x d s \\
& +C\left(\epsilon, T,\left\|u_{0}\right\|_{L^{4}},\left\|\partial_{x}\left(\rho_{0}^{\frac{3}{4}}\right)\right\|_{L^{4}}\right),
\end{aligned}
$$

whence

$$
\begin{aligned}
& \int_{0}^{1}\left\{\partial_{x}\left(\rho^{\frac{3}{4}}\right) r^{2}\right\}^{4} d x \\
& \quad \leq C \int_{0}^{t} \int_{0}^{1}\left(\rho^{\gamma}\right)_{x}^{4}(x, t) d x d s+C\left(\epsilon, T,\left\|u_{0}\right\|_{L^{4}},\left\|\partial_{x}\left(\rho_{0}^{\frac{3}{4}}\right)\right\|_{L^{4}}\right) \\
& \quad \leq C \int_{0}^{t} \max _{[0,1]}\left(\rho^{4 \gamma-3}\right) \int_{0}^{1}\left[\partial_{x}\left(\rho^{\frac{3}{4}}\right)\right]^{4} d x d s+C\left(\epsilon, T,\left\|u_{0}\right\|_{L^{4}},\left\|\partial_{x}\left(\rho_{0}^{\frac{3}{4}}\right)\right\|_{L^{4}}\right) .
\end{aligned}
$$


Then we can get

$$
\int_{0}^{1}\left\{\partial_{x}\left(\rho^{\frac{3}{4}}\right) r^{2}\right\}^{4} d x \leq C
$$

due to Gronwall's inequality and Lemma 4.2. This completes the proof.

Lemma 4.5 There is a positive constant $C=C\left(\epsilon, T,\left\|u_{0}\right\|_{L^{4}},\left\|\left(\rho_{0}^{\frac{3}{4}}\right)_{x}\right\|_{L^{4}}\right)$ such that

$$
\rho \geq C, \quad \forall x \in[0,1], \tau \in[0, T]
$$

Proof Set $v(x, t)=\frac{1}{\rho(x, t)}$ and $V(\tau)=\max _{[0,1] \times[0, \tau]} v(x, s)$. Equation $(4.16)_{1}$ can be rewritten as $v_{\tau}=\left(r^{2} u\right)_{x}$, and then we have

$$
\int_{0}^{\tau} \int_{0}^{1} v_{s} d x d s=\int_{0}^{\tau} \int_{0}^{1}\left(r^{2} u\right)_{x} d x d s
$$

i.e.,

$$
\int_{0}^{1} v(x, \tau) d x=\int_{0}^{1} v_{0}(x) d x=\int_{0}^{1} \frac{1}{\rho_{0}+\epsilon} d x \leq C(\epsilon)
$$

Using Hölder inequality, Lemma 4.4, and Sobolev's embedding $W^{1,1}([0,1]) \hookrightarrow L^{\infty}([0,1])$ yields, for any $0<\beta<1$,

$$
\begin{aligned}
v^{\beta}(x, \tau) & \leq \int_{0}^{1} v^{\beta}(x, \tau) d x+\int_{0}^{1}\left|\partial_{x} v^{\beta}(x, \tau)\right| d x \\
& \leq\left(\int_{0}^{1} v(x, \tau) d x\right)^{\beta}+\frac{4}{3} \beta \int_{0}^{1}\left|v^{\beta+\frac{3}{4}} \partial_{x}\left(\rho^{\frac{3}{4}}\right)\right| d x \\
& \leq C+C \beta\left(\int_{0}^{1}\left(v^{\beta+\frac{3}{4}}\right)^{\frac{4}{3}} d x\right)^{\frac{3}{4}}\left(\int_{0}^{1}\left(\partial_{x}\left(\rho^{\frac{3}{4}}\right)\right)^{4} d x\right)^{\frac{1}{4}} \\
& \leq C+C \beta V^{\beta}\left(\int_{0}^{1} v d x\right)^{\frac{3}{4}}\left(\int_{0}^{1}\left(\partial_{x}\left(\rho^{\frac{3}{4}}\right)\right)^{4} d x\right)^{\frac{1}{4}} \\
& \leq C+C \beta V^{\beta},
\end{aligned}
$$

where $C$ is a constant depending on $\epsilon, T,\left\|u_{0}\right\|_{L^{4}}$, and $\left\|\left(\rho_{0}^{\frac{3}{4}}\right)_{x}\right\|_{L^{4}}$. Taking the supremum on both sides of the above inequality and choosing $\beta>0$ small enough, which may depend on $\epsilon$ and $T$, we obtain

$$
V^{\beta}(T) \leq C\left(\epsilon, T,\left\|u_{0}\right\|_{L^{4}},\left\|\left(\rho_{0}^{\frac{3}{4}}\right)_{x}\right\|_{L^{4}}\right)
$$

Then we have

$$
\rho(x, \tau) \geq \frac{1}{V(x, t)} \geq C\left(\epsilon, T,\left\|u_{0}\right\|_{L^{4}},\left\|\left(\rho_{0}^{\frac{3}{4}}\right)_{x}\right\|_{L^{4}}\right),
$$

and so the lemma is proved. 


\section{Proof of the main result}

In this section, we will prove Theorem 3.4 by completing the constructions of approximate solutions and applying the a priori bounds of Sects. 3 and 4 to take appropriate limits.

\subsection{Global existence of the approximate solutions}

In Sect. 4, we have established the a priori estimates in Lemmas 4.1-4.5. First, we prove the existence of approximate solutions. For the approximate system (4.16)-(4.18), we first regularize the initial data as follows. We denote by $J_{\delta}$ the Friedrichs mollifier of width $\delta$. Let $\left(\rho_{0}+\epsilon, u_{0}\right)$ be the initial data in Eulerian coordinates, where $u_{0}=\frac{m_{0}}{\rho_{0}+\epsilon}$. For simplicity we still denote by $\left(\rho_{0}+\epsilon, u_{0}\right)$ the extension of $\left(\rho_{0}+\epsilon, u_{0}\right)$, i.e.,

$$
\rho_{0}(r)+\epsilon:= \begin{cases}\rho_{0}(\epsilon)+\epsilon, & r \in[0, \epsilon], \\ \rho_{0}(r)+\epsilon, & r \in(\epsilon, r), \\ \rho_{0}(R)+\epsilon, & r \in[R,+\infty],\end{cases}
$$

and

$$
u_{0}(r):= \begin{cases}0, & r \in[0, \epsilon+2 \delta], \\ u_{0}(r), & r \in(\epsilon+2 \delta, R-2 \delta), \\ 0, & r \in[R-2 \delta, R] .\end{cases}
$$

Then we mollify $\left(\rho_{0}(r)+\epsilon, u_{0}(r)\right)$ above with $J_{\delta}$ to get smooth approximate initial data denoted by $\left(\rho_{0}^{\epsilon, \delta}(r), u_{0}^{\epsilon, \delta}(r)\right)$.

Obviously, the resulting data $\left(\rho_{0}^{\epsilon, \delta}(r), u_{0}^{\epsilon, \delta}(r)\right)$ satisfy hypotheses (3.31) (uniformly bounded on $\epsilon$ and $\delta$ ). For any fixed $\epsilon>0$, we denote the corresponding initial data in Lagrangian coordinates by $\left(\rho_{0}^{\delta}, u_{0}^{\delta}\right)$. Then $\rho_{0}^{\delta} \in C^{1+\sigma}[0,1]$ and $u_{0}^{\delta} \in C^{2+\sigma}[0,1]$ for any $0<\sigma<1$. Moreover, as $\delta \rightarrow 0$, we have

$$
\rho_{0}^{\delta} \rightarrow \rho_{0}+\epsilon \quad \text { in } W^{1,2}([0,1]), \quad u_{0}^{\delta} \rightarrow u_{0} \quad \text { in } L^{2}([0,1])
$$

furthermore,

$$
u_{0}^{\delta}(0, \tau)=u_{0}^{\delta}(1, \tau)=0
$$

The short-time existence of a unique classical solution $\left(\rho^{\delta}, u^{\delta}\right)$ to the initial boundary value problem (4.16) with the initial data $\left(\rho_{0}^{\delta}, u_{0}^{\delta}\right)$ and the boundary condition (5.4) can be shown by the standard argument as in [18]. By the a priori estimates established in Lemmas 4.1-4.5 for $\left(\rho^{\delta}, u^{\delta}\right)$ and a continuity argument, we show that it is indeed a global classical solution to (4.16) with the initial data $\left(\rho_{0}^{\delta}, u_{0}^{\delta}\right)$ and the boundary condition (5.4). Then by transforming it into Euler coordinates again, we can obtain the solutions $\left(\rho^{\epsilon, \delta}(r, t), u^{\epsilon, \delta}(r, t)\right)$ to the approximate system (4.2), and consequently Lemma 4.1 holds for these approximate solutions. 


\subsection{Energy and entropy estimates of the approximate solutions}

So far, $\left(\rho^{\epsilon, \delta}, u^{\epsilon, \delta}\right)$ are defined on $\epsilon \leq r \leq R$. To take the limit as $\left\{\epsilon_{j}, \delta_{j}\right\} \rightarrow 0$, we extend $\rho^{\epsilon_{j}, \delta_{j}}(r, t), u^{\epsilon_{j}, \delta_{j}}(r, t)$ to the whole domain $\Omega$ in the following way:

$$
\begin{gathered}
\tilde{\rho}^{\epsilon_{j}, \delta_{j}}= \begin{cases}\rho^{\epsilon_{j}, \delta_{j}}(r, t), & r \in\left[\epsilon_{j}, R\right], \\
\rho^{\epsilon_{j}, \delta_{j}}\left(\epsilon_{j}, t\right), & r \in\left[0, \epsilon_{j}\right],\end{cases} \\
\tilde{u}^{\epsilon_{j}, \delta_{j}}= \begin{cases}u^{\epsilon_{j}, \delta_{j}}(r, t), & r \in\left[\epsilon_{j}, R\right], \\
0, & r \in\left[0, \epsilon_{j}\right],\end{cases}
\end{gathered}
$$

and keep denoting the so-obtained approximate solutions $\left\{\widetilde{\rho}^{\epsilon_{j}, \delta_{j}}, \widetilde{u}^{\epsilon_{j}, \delta_{j}}\right\}$ by $\left\{\rho^{\epsilon_{j}, \delta_{j}}, u^{\epsilon_{j}, \delta_{j}}\right\}$. Let $\rho^{\epsilon_{j}, \delta_{j}}(\mathbf{x}, t)=\rho^{\epsilon_{j}, \delta_{j}}(r, t), U^{\epsilon_{j}, \delta_{j}}(\mathbf{x}, t)=u^{\epsilon_{j}, \delta_{j}}(r, t) \frac{\mathbf{x}}{r}$. For simplicity, we write $\left(\rho^{j}, U^{j}\right)$ instead of $\left(\rho^{\epsilon_{j}, \delta_{j}}, U^{\epsilon_{j}, \delta_{j}}\right)$ and denote $\Omega_{\epsilon}=\Omega \backslash B_{\epsilon}(0)$ for $\epsilon>0$ and $\Omega_{\frac{1}{n}}=\Omega \backslash B_{\frac{1}{n}}(0)$ for $n \in N$, where $N$ is the set of the positive integers. It then follows directly from Lemma 3.1 that

Lemma 5.1 Let $\left(\rho^{j}, U^{j}\right)(x, t)$ be the approximate solutions of (3.1)-(3.3) constructed above. Then there exists a constant $C$ independent of $\epsilon$ such that

$$
\begin{aligned}
& \sup _{t \in[0, T]} \int_{\Omega_{\epsilon_{j}}} \rho^{j}(x, t) d x \leq C, \\
& \sup _{t \in[0, T]} \int_{\Omega_{\epsilon_{j}}}\left\{\frac{1}{2} \rho^{j}\left|U^{j}\right|^{2}+\left|\nabla \phi^{j}\right|^{2}+\frac{1}{\gamma-1}\left(\rho^{j}\right)^{\gamma}\right\} d x+\int_{0}^{T} \int_{\Omega_{\epsilon_{j}}} \rho^{j}\left|\nabla U^{j}\right|^{2} d x d t \\
& \quad+\frac{1}{4} \int_{0}^{T} \int_{\Omega_{\epsilon_{j}}} \epsilon_{j}\left(\rho^{j}\right)^{\frac{3}{4}}\left|\nabla U^{j}\right|^{2} d x d t \leq C, \\
& \quad \sup _{t \in[0, T]} \int_{\Omega_{\epsilon_{j}}} \frac{1}{2} \rho^{j}\left|U^{j}+\nabla \log \rho^{j}+\frac{3}{4} \epsilon_{j}\left(\rho^{j}\right)^{-\frac{5}{4}} \nabla \rho^{j}\right|^{2} d x \\
& \quad+\int_{0}^{T} \int_{\Omega_{\epsilon_{j}}}\left\{\frac{1}{2}\left|\nabla \phi^{j}\right|^{2}+\frac{1}{\gamma-1}\left(\rho^{j}\right)^{\gamma}\right\} d x d t \\
& \quad+\int_{0}^{T} \int_{\Omega_{\epsilon_{j}}} \frac{4}{\gamma}\left|\nabla\left(\rho^{j}\right)^{\frac{\gamma}{2}}\right|^{2} d x d t+\int_{0}^{T} \int_{\Omega_{\epsilon_{j}}} \frac{48 \epsilon \gamma}{(4 \gamma-1)^{2}}\left|\nabla\left(\rho^{j}\right)^{\frac{4 \gamma-1}{8}}\right|^{2} d x d t \leq C .
\end{aligned}
$$

Moreover, the following uniform estimates hold:

$$
\begin{aligned}
& \sup _{t \in[0, T]}\left\|\sqrt{\rho^{j}}\right\|_{H^{1}(\Omega)} \leq C, \\
& \sup _{t \in[0, T]} \int_{\Omega} \rho^{j}\left|U^{j}\right|^{2} d x \leq C .
\end{aligned}
$$

Proof Inequalities (5.7)-(5.9) follow directly from Lemma 4.1. It suffices to prove (5.10) and (5.11).

Note that

$$
\sup _{t \in[0, T]}\left\|\sqrt{\rho^{j}}\right\|_{L^{2}(\Omega)}^{2} \leq \sup _{t \in[0, T]} \int_{0}^{\epsilon_{j}} \rho^{j} r^{2} d r+\sup _{t \in[0, T]} \int_{\epsilon_{j}}^{R} \rho^{j} r^{2} d r
$$




$$
\begin{aligned}
& \leq \frac{C}{\epsilon^{2}} \int_{0}^{\epsilon_{j}} r^{2} d r+C \\
& \leq C
\end{aligned}
$$

due to Lemma 4.1 and Lemma 4.2.

Equation (5.5) gives

$$
\nabla \sqrt{\rho^{j}}(x, t)=0, \quad x \in \bar{B}_{j} .
$$

Thus, using (4.12) and (5.13) yields

$$
\begin{aligned}
\sup _{t \in[0, T]}\left\|\nabla \sqrt{\rho^{j}}\right\|_{L^{2}(\Omega)}^{2} & =\sup _{t \in[0, T]} \int_{\Omega_{\epsilon_{j}}}\left(\frac{1}{2} \frac{\nabla \rho^{j}}{\sqrt{\rho^{j}}}\right)^{2} d x \\
& =\sup _{t \in[0, T]} \frac{1}{4} \int_{\epsilon_{j}}^{R} \frac{\left(\rho_{r}^{j}\right)^{2}}{\rho^{j}} r^{2} d r \\
& \leq C,
\end{aligned}
$$

therefore, combining (5.12) and (5.14), we obtain (5.10).

Using (5.6) and (5.8) yields

$$
\sup _{t \in[0, T]} \int_{\Omega} \rho^{j}\left|U^{j}\right|^{2} d x \leq \sup _{t \in[0, T]} \int_{\Omega_{\epsilon_{j}}} \rho^{j}\left|U^{j}\right|^{2} d x \leq C,
$$

and so the proof of the lemma is completed.

\subsection{Passage to the limit}

Proposition 5.2 There exists a sequence $\left(\epsilon_{j}, \delta_{j}\right)$ and a limiting function $\rho(x, t)$ such that, up to a subsequence, as $j \rightarrow \infty$ we have

$$
\sqrt{\rho^{j}}(\mathbf{x}, t) \rightarrow \sqrt{\rho}(\mathbf{x}, t) \text { a.e. and strongly in } L^{2}\left(0, T ; L^{2}(\Omega)\right),
$$

and

$$
\rho^{j}(\mathbf{x}, t) \rightarrow \rho(\mathbf{x}, t) \quad \text { strongly in } C\left([0, T], L^{\frac{3}{2}}(\Omega)\right) .
$$

Moreover, $\rho(\mathbf{x}, t)=\rho(r, t)$ is a spherically symmetric function.

Proof Equation (5.10) shows that $\sqrt{\rho^{j}}$ is bounded in $L^{\infty}\left(0, T ; H^{1}(\Omega)\right)$. Next we notice that

$$
\partial_{t} \sqrt{\rho^{j}}=-\frac{1}{2} \sqrt{\rho^{j}} \operatorname{div} u^{j}-u^{j} \cdot \nabla \sqrt{\rho^{j}}=\frac{1}{2} \sqrt{\rho^{j}} \operatorname{div} u^{j}-\operatorname{div}\left(u^{j} \sqrt{\rho^{j}}\right) .
$$

Therefore, $\partial_{t} \sqrt{\rho^{j}}$ is bounded in $L^{\infty}\left(0, T ; H^{-1}(\Omega)\right)$ due to Lemma 5.1. Thus (5.16) holds.

Using (5.5), (5.6) and Hölder inequality yields

$$
\left\|\rho^{j} U^{j}\right\|_{L^{\infty}\left(L^{\frac{3}{2}}\right)}^{\frac{3}{2}} \leq \sup _{t \in[0, T]}\left\{\int_{\Omega}\left(\rho^{j}\right)^{3} d x\right\}^{\frac{1}{4}}\left\{\int_{\Omega}\left(\sqrt{\rho^{j}} U^{j}\right)^{2} d x\right\}^{\frac{3}{4}} \leq C,
$$


from which, combining with the fact that $\rho_{t}^{j}=-\operatorname{div}\left(\rho^{j} U^{j}\right)$, we have

$$
\left\|\rho_{t}^{j}\right\|_{L^{\infty}\left(0, T ; W^{-1, \frac{3}{2}}(\Omega)\right)} \leq C
$$

By a similar method, we deduce

$$
\begin{aligned}
\left\|\nabla \rho^{j}\right\|_{L^{\infty}\left(L^{\frac{3}{2}}\right)}^{\frac{3}{2}} & =2 \int_{\Omega} \sqrt{\rho^{j}} \frac{3}{\frac{3}{2}}\left(\nabla \sqrt{\rho^{j}}\right)^{\frac{3}{2}} d x \\
& \leq 2\left(\int_{\Omega}\left(\rho^{j}\right)^{3} d x\right)^{\frac{1}{4}}\left(\int_{\Omega}\left(\nabla \sqrt{\rho^{j}}\right)^{2} d x\right)^{\frac{3}{4}} \\
& \leq C .
\end{aligned}
$$

It follows from (5.10) and (5.21) that

$$
\left\|\rho^{j}\right\|_{L^{\infty}\left(0, T ; W^{1, \frac{3}{2}}(\Omega)\right)} \leq C
$$

which combined with (5.20) yields

$$
\left\{\rho^{j}\right\}_{j=1}^{\infty} \text { is precompact in } C\left([0, T] ; W^{1, \frac{3}{2}}-w\right) \text {. }
$$

Thus, we get

$$
\rho^{j}(x, t) \rightarrow \rho(x, t) \quad \text { strongly in } C\left([0, T], L^{\frac{3}{2}}(\Omega)\right),
$$

since $W^{1, \frac{3}{2}}(\Omega) \hookrightarrow \hookrightarrow L^{\frac{3}{2}}(\Omega)$. The proof of this proposition is completed.

Proposition 5.3 Suppose $1<\gamma<3$. Then $\left(\rho^{j}\right)^{\gamma}$ converges to $\rho^{\gamma}$ in $L^{1}\left((0, T), L^{1}(\Omega)\right)$.

Proof It follows from the fact that $\left\|\rho^{j}\right\|_{L^{\infty}\left(L^{3}(\Omega)\right)}$ is bounded and $\rho^{j}(x, t) \rightarrow \rho(x, t)$ strongly in $L^{\infty}\left([0, T] ; L^{\frac{3}{2}}(\Omega)\right)$ that

$$
\rho^{j}(x, t) \longrightarrow \rho(x, t) \quad \text { strongly in } L^{\infty}\left([0, T] ; L^{p}(\Omega)\right), \frac{3}{2} \leq p<3 .
$$

Since $\Omega$ is bounded,

$$
\rho^{j}(x, t) \longrightarrow \rho(x, t) \quad \text { strongly in } L^{\infty}\left([0, T] ; L^{\gamma}(\Omega)\right), 1<\gamma<3
$$

Applying Hölder inequality and (5.26), we have

$$
\begin{aligned}
\int_{\Omega}\left|\left(\rho^{j}\right)^{\gamma}-\rho^{\gamma}\right| d x & \leq \int_{\Omega}\left|\left(\rho^{j}-\rho\right) \max \left\{\left(\rho^{j}\right)^{\gamma-1}, \rho^{\gamma-1}\right\}\right| d x \\
& \leq\left(\int_{\Omega}\left|\rho^{j}-\rho\right|^{\gamma} d x\right)^{\frac{1}{\gamma}}\left(\int_{\Omega} \max \left\{\left(\rho^{j}\right)^{\gamma-1}, \rho^{\gamma-1}\right\}^{\frac{\gamma}{\gamma-1}} d x\right)^{\frac{\gamma-1}{\gamma}} \\
& \rightarrow 0, \quad \text { as } j \rightarrow \infty,
\end{aligned}
$$

i.e., $\left(\rho^{j}\right)^{\gamma} \rightarrow \rho^{\gamma}$ strongly in $L^{\infty}\left(L^{1}(\Omega)\right)$. This proves the proposition. 
Lemma 5.4 The pressure $\left(\rho^{j}\right)^{\gamma}$ is bounded in $L^{\frac{5}{3}}\left((0, T), L^{\frac{5}{3}}\left(\Omega_{\epsilon_{j}}\right)\right)$.

Proof Lemma 5.1 shows that

$$
\left(\rho^{j}\right)^{\gamma / 2} \in L^{2}\left(0, T ; H^{1}\left(\Omega \epsilon_{j}\right)\right) \text { and }\left(\rho^{j}\right)^{\gamma} \in L^{1}\left(0, T ; L^{3}\left(\Omega \epsilon_{j}\right)\right)
$$

Since $\left(\rho^{j}\right)^{\gamma}$ is bounded in $L^{\infty}\left(0, T ; L^{1}\left(\Omega \epsilon_{j}\right)\right)$ by (5.8), using Hölder inequality, we have

$$
\begin{aligned}
\left\|\left(\rho^{j}\right)^{\gamma}\right\|_{L^{5 / 3}\left(L^{5 / 3}\left(\Omega \epsilon_{j}\right)\right)}^{5 / 3} & \leq \int_{0}^{T}\left(\int_{\Omega \epsilon_{j}}\left(\rho^{j}\right)^{\gamma} d x\right)^{\frac{2}{3}}\left(\int_{\Omega \epsilon_{j}}\left(\rho^{j}\right)^{3 \gamma} d x\right)^{\frac{1}{3}} d t \\
& \leq\left\|\left(\rho^{j}\right)^{\gamma}\right\|_{L^{\infty}\left(L^{1}\left(\Omega \epsilon_{j}\right)\right)}^{\frac{2}{3}}\left\|\left(\rho^{j}\right)^{\gamma}\right\|_{L^{1}\left(L^{3}\left(\Omega \epsilon_{j}\right)\right)} \\
& \leq C
\end{aligned}
$$

where $C$ is independent of $\epsilon_{j}$. The proof of the lemma is completed.

Proposition 5.5 If $1<\gamma<3$ and $\int_{0}^{R} \rho_{0}\left|u_{0}\right|^{2} r^{2} d r \leq C$, then the following inequality holds:

$$
\begin{gathered}
\int_{\epsilon_{j}}^{R} \rho^{j} \frac{\left|u^{j}\right|^{2+\eta}}{2+\eta} r^{2} d r+\int_{0}^{t} \int_{\epsilon_{j}}^{R}\left(\frac{3}{4} \rho^{j}+\frac{\epsilon_{j}}{8}\left(\rho^{j}\right)^{\frac{3}{4}}\right)\left|u^{j}\right|^{\eta}\left(u_{r}^{j}\right)^{2} r^{2} d r d t \\
+\int_{0}^{t} \int_{\epsilon_{j}}^{R}\left(\frac{7}{4} \rho^{j}+\frac{3 \epsilon_{j}}{8}\left(\rho^{j}\right)^{\frac{3}{4}}\right)\left|u^{j}\right|^{\eta+2} d r d t \leq C
\end{gathered}
$$

for small $\eta \in(0,1)$ and $C$ being a constant independent of $\epsilon_{j}$.

Proof Letting $0<\eta<\frac{1}{2}$, multiplying $(4.2)_{2}$ by $r^{2} u^{j}\left|u^{j}\right|^{\eta}$, then integrating the resulting equation, one gets

$$
\begin{aligned}
& \int_{\epsilon_{j}}^{R} \rho^{j} u_{t}^{j} u^{j}\left|u^{j}\right|^{\eta} r^{2} d r+\int_{\epsilon^{j}}^{R} \rho_{t}^{j}\left|u^{j}\right|^{2+\eta} r^{2} d r+\int_{\epsilon_{j}}^{R}\left(\rho^{j}\left(u^{j}\right)^{2}+\left(\rho^{j}\right)^{\gamma}\right)_{r} u^{j}\left|u^{j}\right|^{\eta} r^{2} d r \\
& \quad+\int_{\epsilon_{j}}^{R} 2 \rho^{j} u^{j}\left|u^{j}\right|^{2+\eta} r d r+\int_{\epsilon_{j}}^{R} 2\left|u^{j}\right|^{2+\eta}\left(\rho^{j}+\epsilon_{j}\left(\rho^{j}\right)^{\frac{3}{4}}\right)_{r} r d r \\
& =\int_{\epsilon_{j}}^{R}\left\{\left(\rho^{j}+\frac{3}{4} \epsilon_{j}\left(\rho^{j}\right)^{\frac{3}{4}}\right)\left(u_{r}^{j}+\frac{2 u^{j}}{r}\right)\right\} u_{r}^{j}\left|u^{j}\right|^{\eta} r^{2} d r+\int_{\epsilon_{j}}^{R} \rho^{j} \phi_{r}^{j} u^{j}\left|u^{j}\right|^{\eta} r^{2} d r .
\end{aligned}
$$

With the help of (4.2) 1 and integration by parts, we deduce

$$
\begin{aligned}
& \int_{\epsilon_{j}}^{R} \rho^{j} \partial_{t}\left(\frac{\left|u^{j}\right|^{2+\eta}}{2+\eta}\right) r^{2} d r+\int_{\epsilon_{j}}^{R} \rho^{j} u^{j} \partial_{r}\left(\frac{\left|u^{j}\right|^{2+\eta}}{2+\eta}\right) r^{2} d r+\int_{\epsilon_{j}}^{R}\left(\left(\rho^{j}\right)^{\gamma}\right)_{r} u^{j}\left|u^{j}\right|^{\eta} r^{2} d r \\
& \quad+(1+\eta) \int_{\epsilon_{j}}^{R}\left(\rho^{j}+\frac{3}{4} \epsilon_{j}\left(\rho^{j}\right)^{\frac{3}{4}}\right)\left(u_{r}^{j}\right)^{2}\left|u^{j}\right|^{\eta} r^{2} d r+\int_{\epsilon_{j}}^{R}\left(2 \rho^{j}+\epsilon_{j}\left(\rho^{j}\right)^{\frac{3}{4}}\right)\left|u^{j}\right|^{2+\eta} d r \\
& =\left(1+\frac{\eta}{2}\right) \int_{\epsilon_{j}}^{R} \epsilon_{j}\left(\rho^{j}\right)^{\frac{3}{4}} u^{j}\left|u^{j}\right|^{\eta} u_{r}^{j} r d r+\int_{\epsilon_{j}}^{R} \rho^{j} \phi_{r}^{j} u^{j}\left|u^{j}\right|^{\eta} r^{2} d r .
\end{aligned}
$$


Then using Cauchy inequality gives

$$
\begin{aligned}
& \int_{\epsilon_{j}}^{R} \rho^{j} \partial_{t}\left(\frac{\left|u^{j}\right|^{2+\eta}}{2+\eta}\right) r^{2} d r+\int_{\epsilon_{j}}^{R} \rho^{j} u^{j} \partial_{r}\left(\frac{\left|u^{j}\right|^{2+\eta}}{2+\eta}\right) r^{2} d r+\int_{\epsilon_{j}}^{R}\left(\left(\rho^{j}\right)^{\gamma}\right)_{r} u^{j}\left|u^{j}\right|^{\eta} r^{2} d r \\
& \quad+\int_{\epsilon_{j}}^{R}\left(\rho^{j}+\frac{\epsilon_{j}}{8}\left(\rho^{j}\right)^{\frac{3}{4}}\right)\left(u_{r}^{j}\right)^{2}\left|u^{j}\right|^{\eta} r^{2} d r+\int_{\epsilon_{j}}^{R}\left(2 \rho^{j}+\frac{3}{8} \epsilon_{j}\left(\rho^{j}\right)^{\frac{3}{4}}\right)\left|u^{j}\right|^{2+\eta} d r \\
& \leq \int_{\epsilon_{j}}^{R} \rho^{j} \phi_{r}^{j} u^{j}\left|u^{j}\right|^{\eta} r^{2} d r .
\end{aligned}
$$

Multiplying (4.2) $)_{1}$ by $\frac{r^{2}\left|u^{j}\right|^{2+\eta}}{2+\eta}$ and integrating by parts yields

$$
\int_{\epsilon_{j}}^{R} \frac{\left|u^{j}\right|^{2+\eta}}{2+\eta} \rho_{t}^{j} r^{2} d r-\int_{\epsilon_{j}}^{R} \rho^{j} u^{j}\left(\frac{\left|u^{j}\right|^{2+\eta}}{2+\eta}\right)_{r} r^{2} d r=0 .
$$

Adding the last two inequalities gives

$$
\begin{aligned}
& \frac{d}{d t} \int_{\epsilon_{j}}^{R} \frac{\left|u^{j}\right|^{2+\eta}}{2+\eta} \rho^{j} r^{2} d r+\int_{\epsilon_{j}}^{R}\left(\rho^{j}+\frac{1}{8} \epsilon_{j}\left(\rho^{j}\right)^{\frac{3}{4}}\right)\left(u_{r}^{j}\right)^{2}\left|u^{j}\right|^{\eta} r^{2} d r \\
& \quad+\int_{\epsilon_{j}}^{R}\left\{2 \rho^{j}+\frac{3}{8} \epsilon_{j}\left(\rho^{j}\right)^{\frac{3}{4}}\right\}\left|u^{j}\right|^{2+\eta} d r \\
& \leq\left.\left|\int_{\epsilon_{j}}^{R}\left[\left(\rho^{j}\right)^{\gamma}\right]_{r} u^{j}\right| u^{j}\right|^{\eta} r^{2} d r|+| \int_{\epsilon_{j}}^{R} \rho^{j} \phi_{r}^{j} u^{j}\left|u^{j}\right|^{\eta} r^{2} d r \mid .
\end{aligned}
$$

It follows from Hölder and Young inequalities that the first term on the right-hand side of (5.34) is estimated as follows:

$$
\begin{aligned}
& \left.\left|\int_{\epsilon_{j}}^{R}\left[\left(\rho^{j}\right)^{\gamma}\right]_{r} u^{j}\right| u^{j}\right|^{\eta} r^{2} d r \mid \\
& =\left|-\int_{\epsilon_{j}}^{R}\left(\rho^{j}\right)^{\gamma}\left(u^{j}\left|u^{j}\right|^{\eta} r^{2}\right)_{r} d r\right| \\
& \leq(1+\eta)\left(\int_{\epsilon_{j}}^{R} \rho^{j}\left|u^{j}\right|^{\eta}\left|u_{r}^{j}\right|^{2} r^{2} d r\right)^{\frac{1}{2}}\left(\int_{\epsilon_{j}}^{R}\left(\rho^{j}\right)^{2 \gamma-1}\left|u^{j}\right|^{\eta} r^{2} d r\right)^{\frac{1}{2}} \\
& \quad+2 \int_{\epsilon_{j}}^{R}\left|u^{j}\right|^{\eta+1}\left(\rho^{j}\right)^{\gamma} r d r \\
& \leq \frac{1}{4} \int_{\epsilon_{j}}^{R} \rho^{j}\left|u^{j}\right|^{\eta}\left|u_{r}^{j}\right|^{2} r^{2} d r+C \int_{\epsilon_{j}}^{R}\left(\rho^{j}\right)^{2 \gamma-1}\left|u^{j}\right|^{\eta} r^{2} d r \\
& \quad+2 \int_{\epsilon_{j}}^{R}\left|u^{j}\right|^{\eta+1}\left(\rho^{j}\right)^{\gamma} r d r,
\end{aligned}
$$

where constant $C>0$ is independent of $\epsilon_{j}$. 
Now it remains to bound the last two terms on the right-hand side of (5.35). Using Lemma 5.1 and Hölder inequality yields

$$
\begin{aligned}
\int_{\epsilon_{j}}^{R}\left(\rho^{j}\right)^{2 \gamma-1}\left|u^{j}\right|^{\eta} r^{2} d r & \leq\left(\int_{\epsilon_{j}}^{R} \rho^{j}\left|u^{j}\right|^{2} r^{2} d r\right)^{\frac{\eta}{2}}\left(\int_{\epsilon_{j}}^{R}\left(\rho^{j}\right)^{\frac{2}{2-\eta}\left(2 \gamma-1-\frac{\eta}{2}\right)} r^{2} d r\right)^{\frac{2-\eta}{2}} \\
& \leq C \int_{\epsilon_{j}}^{R}\left(\rho^{j}\right)^{\frac{2}{2-\eta}\left(2 \gamma-1-\frac{\eta}{2}\right)} r^{2} d r+C, \\
\int_{\epsilon_{j}}^{R}\left|u^{j}\right|^{\eta+1}\left(\rho^{j}\right)^{\gamma} r d r & \leq\left(\int_{\epsilon_{j}}^{R} \rho^{j}\left|u^{j}\right|^{\eta+2} d r\right)^{\frac{1+\eta}{2+\eta}}\left(\int_{\epsilon_{j}}^{R}\left(\rho^{j}\right)^{\left(\gamma-\frac{1+\eta}{2+\eta}\right)(2+\eta)} r^{2+\eta} d r\right)^{\frac{1}{2+\eta}} \\
& \leq \frac{1}{4} \int_{\epsilon_{j}}^{R} \rho^{j}\left|u^{j}\right|^{\eta+2} d r+C(R) \int_{\epsilon_{j}}^{R}\left(\rho^{j}\right)^{\left(\gamma-\frac{1+\eta}{2+\eta}\right)(2+\eta)} r^{2} d r .
\end{aligned}
$$

Substituting the above two estimates into (5.35) yields

$$
\begin{aligned}
& \left.\left|\int_{\epsilon_{j}}^{R}\left(\left(\rho^{j}\right)^{\gamma}\right)_{r} u^{j}\right| u^{j}\right|^{\eta} r^{2} d r \mid \\
& \quad \leq \frac{1}{4} \int_{\epsilon_{j}}^{R} \rho^{j}\left|u^{j}\right|^{\eta}\left|u_{r}^{j}\right|^{2} r^{2} d r+C \int_{\epsilon_{j}}^{R}\left(\rho^{j}\right)^{\frac{2}{2-\eta}\left(2 \gamma-1-\frac{\eta}{2}\right)} r^{2} d r+\frac{1}{4} \int_{\epsilon_{j}}^{R} \rho^{j}\left|u^{j}\right|^{\eta+2} d r \\
& \quad+C \int_{\epsilon_{j}}^{R}\left(\rho^{j}\right)^{\left(\gamma-\frac{1+\eta}{2+\eta}\right)(2+\eta)} r^{2} d r+C .
\end{aligned}
$$

Then using Hölder and Young inequalities, as well as the fact

$$
\int_{\Omega_{\epsilon_{j}}}\left|\nabla \phi^{j}\right|^{3+\frac{3}{2} \eta} d x \leq C, \quad \text { when } 0<\eta<\frac{1}{2}
$$

which will be proved later, the second term on the right-hand side of (5.34) can be estimated as follows:

$$
\begin{aligned}
& \left.\left|\int_{\epsilon_{j}}^{R} \rho^{j} \phi_{r}^{j} u^{j}\right| u^{j}\right|^{\eta} r^{2} d r \mid \\
& \quad \leq \int_{\Omega_{\epsilon_{j}}} \rho^{j}\left|\nabla \phi^{j}\right|\left|U^{j}\right|^{\eta+1} d x \\
& \quad \leq C \int_{\Omega_{\epsilon_{j}}} \rho^{j}\left|U^{j}\right|^{\eta+2} d x+C \int_{\Omega_{\epsilon_{j}}}\left|\nabla \phi^{j}\right|^{\eta+2} \rho^{j} d x \\
& \quad \leq C \int_{\Omega_{\epsilon_{j}}} \rho^{j}\left|U^{j}\right|^{\eta+2} d x+C\left(\int_{\Omega_{\epsilon_{j}}}\left|\nabla \phi^{j}\right|^{3+\frac{3}{2} \eta} d x\right)^{\frac{2}{3}}\left(\int_{\Omega_{\epsilon_{j}}}\left(\rho^{j}\right)^{3} d x\right)^{\frac{1}{3}} \\
& \quad \leq C+C \int_{\Omega_{\epsilon_{j}}} \rho^{j}\left|U^{j}\right|^{\eta+2} d x \\
& \quad=C+C \int_{\epsilon_{j}}^{R} \rho^{j}\left|u^{j}\right|^{\eta+2} r^{2} d r .
\end{aligned}
$$


Now we give the proof of (5.39). Lemma 5.1 shows that $\rho^{j}$ is bounded in $L^{\infty}\left(L^{3}(\Omega)\right)$. Using the regularity theory of elliptic equations, we have

$$
\left\|\phi^{j}\right\|_{H^{2}\left(\Omega_{\epsilon_{j}}\right)} \leq C\left(\left\|\phi^{j}\right\|_{H^{1}\left(\Omega_{\epsilon_{j}}\right)}+\left\|\rho^{j}-b\right\|_{L^{2}\left(\Omega_{\epsilon_{j}}\right)}\right)
$$

It follows from Hardy-Littlewood-Sobolev embedding theorem that

$$
\left\|\phi^{j}\right\|_{L^{p^{\prime}}\left(\Omega_{\epsilon_{j}}\right)} \leq C\left(q^{\prime}\right)\left\|\rho^{j}-b\right\|_{L^{q^{\prime}}\left(\Omega_{\epsilon_{j}}\right)}
$$

where $\frac{1}{q^{\prime}}=\frac{1}{p^{\prime}}+\frac{2}{3}$. Letting $p^{\prime}=2$ in (5.42), we get $q^{\prime}=\frac{7}{6}$. Obviously, $\left\|\rho^{j}-b\right\|_{L^{\frac{7}{6}}\left(\Omega_{\epsilon_{j}}\right)} \leq C$, which means that

$$
\left\|\phi^{j}\right\|_{L^{2}\left(\Omega_{\epsilon_{j}}\right)} \leq C
$$

so, combining with (5.8), one gets

$$
\left\|\phi^{j}\right\|_{H^{1}\left(\Omega_{\epsilon_{j}}\right)} \leq C
$$

Returning to (5.41), we obtain

$$
\left\|\phi^{j}\right\|_{H^{2}\left(\Omega_{\epsilon_{j}}\right)} \leq C
$$

which implies that (5.39) holds.

Substituting (5.38) and (5.40) into (5.34), we have

$$
\begin{aligned}
& \frac{d}{d t} \int_{\epsilon_{j}}^{R} \frac{\left|u^{j}\right|^{2+\eta}}{2+\eta} \rho^{j} r^{2} d r+\int_{\epsilon_{j}}^{R}\left(\frac{3}{4} \rho^{j}+\frac{1}{8} \epsilon_{j}\left(\rho^{j}\right)^{\frac{3}{4}}\right)\left(u_{r}^{j}\right)^{2}\left|u^{j}\right|^{\eta} r^{2} d r \\
& \quad+\int_{\epsilon_{j}}^{R}\left(\frac{7}{4} \rho^{j}+\frac{3}{8} \epsilon_{j}\left(\rho^{j}\right)^{\frac{3}{4}}\right)\left|u^{j}\right|^{2+\eta} d r \\
& \leq C \int_{\epsilon_{j}}^{R}\left(\rho^{j}\right)^{\frac{2}{2-\eta}\left(2 \gamma-1-\frac{\eta}{2}\right)} r^{2} d r+C \int_{\epsilon_{j}}^{R}\left(\rho^{j}\right)^{\left(\gamma-\frac{1+\eta}{2+\eta}\right)(2+\eta)} r^{2} d r \\
& \quad+C \int_{\epsilon_{j}}^{R} \rho^{j}\left|u^{j}\right|^{\eta+2} r^{2} d r+C .
\end{aligned}
$$

Integrating both sides of (5.46) with respect to $t$ yields

$$
\begin{aligned}
\int_{\epsilon_{j}}^{R} & \frac{\left|u^{j}\right|^{2+\eta}}{2+\eta} \rho^{j} r^{2} d r+\int_{0}^{t} \int_{\epsilon_{j}}^{R}\left(\frac{3}{4} \rho^{j}+\frac{1}{8} \epsilon_{j}\left(\rho^{j}\right)^{\frac{3}{4}}\right)\left(u_{r}^{j}\right)^{2}\left|u^{j}\right|^{\eta} r^{2} d r d t \\
& +\int_{0}^{t} \int_{\epsilon_{j}}^{R}\left(\frac{7}{4} \rho^{j}+\frac{3}{8} \epsilon_{j}\left(\rho^{j}\right)^{\frac{3}{4}}\right)\left|u^{j}\right|^{2+\eta} d r d t \\
\leq & C \int_{0}^{t} \int_{\epsilon_{j}}^{R}\left(\rho^{j}\right)^{\frac{2}{2-\eta}\left(2 \gamma-1-\frac{\eta}{2}\right)} r^{2} d r d t+C \int_{0}^{t} \int_{\epsilon_{j}}^{R}\left(\rho^{j}\right)^{\left(\gamma-\frac{1+\eta}{2+\eta}\right)(2+\eta)} r^{2} d r d t \\
& +C \int_{0}^{t} \int_{\epsilon_{j}}^{R} \rho^{j}\left|u^{j}\right|^{\eta+2} r^{2} d r d t+C(T) .
\end{aligned}
$$


It follows from Lemma 5.4 that $\left(\rho^{j}\right)^{\gamma}$ is bounded in $L^{\frac{5}{3}}\left((0, T) ; L^{\frac{5}{3}}\left(\Omega_{\epsilon_{j}}\right)\right)$, thus the first and second terms on the right-hand side of (5.47) are bounded when the following inequalities both hold:

$$
\frac{2}{2-\eta}\left(2 \gamma-1-\frac{\eta}{2}\right) \leq \frac{5}{3} \gamma, \quad\left(\gamma-\frac{1+\eta}{2+\eta}\right)(2+\eta) \leq \frac{5}{3} \gamma
$$

which it means that

$$
\int_{0}^{t} \int_{\epsilon_{j}}^{R}\left(\rho^{j}\right)^{\frac{2}{2-\eta}\left(2 \gamma-1-\frac{\eta}{2}\right)} r^{2} d r d t \leq C \quad \text { and } \quad \int_{0}^{t} \int_{\epsilon_{j}}^{R}\left(\rho^{j}\right)^{\left(\gamma-\frac{1+\eta}{2+\eta}\right)(2+\eta)} r^{2} d r d t \leq C
$$

hold, provided that $1<\gamma<3$.

Combining all the arguments above, we obtain

$$
\begin{gathered}
\int_{\epsilon_{j}}^{R} \frac{\left|u^{j}\right|^{2+\eta}}{2+\eta} \rho^{j} r^{2} d r+\int_{0}^{t} \int_{\epsilon_{j}}^{R}\left(\frac{3}{4} \rho^{j}+\frac{1}{8} \epsilon_{j}\left(\rho^{j}\right)^{\frac{3}{4}}\right)\left(u_{r}^{j}\right)^{2}\left|u^{j}\right|^{\eta} r^{2} d r d t \\
\quad+\int_{0}^{t} \int_{\epsilon_{j}}^{R}\left(\frac{7}{4} \rho^{j}+\frac{3}{8} \epsilon_{j}\left(\rho^{j}\right)^{\frac{3}{4}}\right)\left|u^{j}\right|^{2+\eta} d r d t \\
\leq C \int_{0}^{t} \int_{\epsilon_{j}}^{R} \rho^{j}\left|u^{j}\right|^{\eta+2} r^{2} d r d t+C(T) .
\end{gathered}
$$

An application of Gronwall's inequality to (5.50) yields immediately that

$$
\int_{\epsilon_{j}}^{R} \rho^{j}\left|u^{j}\right|^{\eta+2} r^{2} d r \leq C(T)
$$

hence

$$
\int_{0}^{t} \int_{\epsilon_{j}}^{R} \rho^{j}\left|u^{j}\right|^{\eta+2} r^{2} d r d t \leq C(T)
$$

The proof of this proposition is completed.

Lemma 5.6 If $1<\gamma<3$, then $\sqrt{\rho^{j}} U^{j}$ is bounded in $L^{\infty}\left(0, T ; L^{2+2 \xi}(\Omega)\right)$ for some small $\xi>0$.

Proof It follows from (5.6) and (5.29) that

$$
\int_{\Omega} \rho^{j} \frac{\left|u^{j}\right|^{2+\eta}}{2+\eta} d x \leq C
$$

hence

$$
\begin{aligned}
\int_{\Omega}\left(\rho^{j}\left|U^{j}\right|^{2}\right)^{1+\xi} d x & \leq\left(\int_{\Omega} \rho^{j}\left|U^{j}\right|^{2+\eta} d x\right)^{\frac{2+2 \xi}{2+\eta}}\left(\int_{\Omega}\left(\rho^{j}\right)^{1+\frac{(2+\eta) \xi}{\eta-2 \xi}} d x\right)^{\frac{\eta-2 \xi}{2+\eta}} \\
& \leq C\left(\int_{\Omega}\left(\rho^{j}\right)^{1+\frac{(2+\eta) \xi}{\eta-2 \xi}} d x\right)^{\frac{\eta-2 \xi}{2+\eta}}
\end{aligned}
$$


Equation (5.10) implies that $\rho^{j} \in L^{\infty}\left(L^{3}(\Omega)\right)$. Now letting $1+\frac{(2+\eta) \xi}{\eta-2 \xi} \leq 3$, we get

$$
\int_{\Omega}\left(\rho^{j}\right)^{1+\frac{(2+\eta) \xi}{\eta-2 \xi}} d x \leq C
$$

whenever $0<\xi \leq \frac{2 \eta}{6+\eta}$, i.e., the lemma is proved.

\section{Proposition 5.7}

(1) Up to a subsequence, $\mathbf{m}^{j}=\rho^{j} U^{j}$ converges strongly in $L^{2}\left((0, T) ; L^{1+\beta}\left(\Omega_{\frac{1}{n}}\right)\right)$ and almost everywhere to some $\mathbf{m}(x, t)$ for any positive integer $n$ and some $0<\beta<\xi$.

(2) $\sqrt{\rho^{j}} U^{j}$ converges strongly in $L^{2}\left((0, T) \times \Omega_{\frac{1}{n}}\right)$ and almost everywhere to $\frac{\mathbf{m}}{\sqrt{\rho}}$ (defined to be zero when $m=0$ ) for any positive integer $n$. In particular, $\mathbf{m}(x, t)=0$ a.e. on $\{\rho(x, t)=0\}$, and there exists a function $U(x, t)$ such that

$$
\mathbf{m}(x, t)=\rho(x, t) U(x, t)
$$

Proof (1) It can be easily obtained that

$$
\rho^{j} U^{j}=\sqrt{\rho^{j}} \sqrt{\rho^{j}} U^{j} \in L^{\infty}\left(L^{\frac{3}{2}}\left(\Omega_{\epsilon_{j}}\right)\right),
$$

due to Lemma 5.1 and Hölder inequality.

Furthermore, since $\nabla\left(\rho^{j} U^{j}\right)=\sqrt{\rho^{j}} \sqrt{\rho^{j}} \nabla U^{j}+2 \sqrt{\rho^{j}} U^{j} \nabla \sqrt{\rho^{j}}$, for any $0<\beta<\xi$, we have

$$
\sqrt{\rho^{j}} \sqrt{\rho^{j}} \nabla U^{j} \in L^{2}\left(L^{\frac{3}{2}}\left(\Omega_{\epsilon_{j}}\right)\right)
$$

and

$$
\sqrt{\rho^{j}} U^{j} \nabla \sqrt{\rho^{j}} \in L^{\infty}\left(L^{1+\beta}(\Omega),\right.
$$

due to Lemmas 5.1, 5.6 and Hölder inequality. Hence, we get

$$
\nabla\left(\rho^{j} U^{j}\right) \in L^{2}\left(L^{1+\beta}\left(\Omega_{\epsilon_{j}}\right)\right)
$$

Equations (5.56) and (5.59) show that

$$
\rho^{j} U^{j} \in L^{2}\left(W^{1,1+\beta}\left(\Omega_{\epsilon_{j}}\right)\right)
$$

Moreover,

$$
\begin{aligned}
\left(\rho^{j} U^{j}\right)_{t}= & \rho^{j} \nabla \Phi^{j}-\operatorname{div}\left(\rho^{j} U^{j} \otimes U^{j}\right)+\operatorname{div}\left(\left(\rho^{j}+\epsilon_{j}\left(\rho^{j}\right)^{\frac{3}{4}}\right) \nabla U^{j}\right) \\
& -\nabla\left(\frac{\epsilon_{j}}{4}\left(\rho^{j}\right)^{\frac{3}{4}} \operatorname{div} U^{j}\right)-\nabla\left(\rho^{j}\right)^{\gamma}
\end{aligned}
$$

Lemma 5.1 and (5.45) imply that

$$
\rho^{j} \nabla \Phi^{j} \in L^{\infty}\left(L^{2}\left(\Omega_{\epsilon_{j}}\right)\right) \hookrightarrow L^{\infty}\left(W^{-1,1+\beta}\left(\Omega_{\epsilon_{j}}\right)\right) .
$$


Lemma 5.6 gives that $\sqrt{\rho^{j}} U^{j} \otimes \sqrt{\rho^{j}} U^{j} \in L^{\infty}\left(0, T ; L^{1+\xi}(\Omega)\right)$. Thus we get $\operatorname{div}\left(\sqrt{\rho^{j}} U^{j} \otimes\right.$ $\left.\sqrt{\rho^{j}} U^{j}\right) \in L^{\infty}\left(W^{-1,1+\xi}\left(\Omega_{\epsilon_{j}}\right)\right) \hookrightarrow L^{\infty}\left(W^{-1,1+\beta}\left(\Omega_{\epsilon_{j}}\right)\right)$ (when $\beta<\xi$ ).

Next we check that $\operatorname{div}\left(\left(\rho^{j}+\epsilon_{j}\left(\rho^{j}\right)^{\frac{3}{4}}\right) \nabla U^{j}\right)$ and $\nabla\left(\epsilon_{j}\left(\rho^{j}\right)^{\frac{3}{4}} \operatorname{div} U^{j}\right)$ are uniformly bounded in $L^{2}\left(W^{-1,1+\beta}\left(\Omega_{\epsilon_{j}}\right)\right)$.

Due to (5.57) and the fact

$$
\left(\rho^{j}\right)^{\frac{3}{4}} \nabla U^{j}=\left(\rho^{j}\right)^{\frac{1}{4}} \sqrt{\rho^{j}} \nabla U^{j} \in L^{2}\left(L^{\frac{3}{2}}\left(\Omega_{\epsilon_{j}}\right)\right),
$$

we deduce

$$
\operatorname{div}\left(\left(\rho^{j}+\epsilon_{j}\left(\rho^{j}\right)^{\frac{3}{4}}\right) \nabla U^{j}\right) \in L^{2}\left(W^{-1, \frac{3}{2}}\left(\Omega_{\epsilon_{j}}\right)\right) \hookrightarrow L^{2}\left(W^{-1,1+\beta}\left(\Omega_{\epsilon_{j}}\right)\right) .
$$

Similarly, we have

$$
\nabla\left(\frac{3}{4}\left(\rho^{j}\right)^{\frac{3}{4}} \operatorname{div} U^{j}\right) \in L^{2}\left(W^{-1,1+\beta}\left(\Omega_{\epsilon_{j}}\right)\right)
$$

It follows from Lemma 5.4 that $\nabla\left(\rho^{j}\right)^{\gamma} \in L^{\frac{5}{3}}\left(W^{-1, \frac{5}{3}}\left(\Omega_{\epsilon_{j}}\right)\right)$. Returning to (5.61), we can get

$$
\left(\rho^{j} U^{j}\right)_{t} \in L^{\frac{5}{3}}\left(W^{-1,1+\beta}\left(\Omega_{\epsilon_{j}}\right)\right)
$$

Furthermore, $W^{1,1+\beta}\left(\Omega_{\epsilon_{j}}\right) \hookrightarrow \hookrightarrow L^{1+\beta}\left(\Omega_{\epsilon_{j}}\right) \hookrightarrow W^{-1,1+\beta}\left(\Omega_{\epsilon_{j}}\right)$. So according to LionsAubin's Lemma, we obtain the compactness of $\mathbf{m}^{j}=\rho^{j} U^{j}$ in $L^{2}\left(0, T ; L^{1+\beta}\left(\Omega_{\frac{1}{n}}\right)\right)$ for all $n \in N^{+}$, i.e., there exists a function $\mathbf{m}(x, t)$ such that for any positive integer $n$ and some $0<\beta<\xi$, it holds that

$$
\mathbf{m}^{j}=\rho^{j} U^{j} \rightarrow \mathbf{m} \quad \text { strongly in } L^{2}\left(0, T ; L^{1+\beta}\left(\Omega_{\frac{1}{n}}\right)\right)
$$

(2) From the proof of (1), if we define $\frac{\mathbf{m}^{2}}{\rho}$ to be zero when $\mathbf{m}=0$, we have $\mathbf{m}(\mathbf{x}, t)=0$ a.e. whenever $\rho(\mathbf{x}, t)=0$. Since $\frac{\mathbf{m}^{j}}{\sqrt{\rho^{j}}}$ is uniformly bounded in $L^{\infty}\left(0, T ; L^{2}\left(\Omega_{\epsilon_{j}}\right)\right)$ and hence in $L^{\infty}\left(0, T ; L^{2}\left(\Omega_{\frac{1}{n}}\right)\right)$ for any $n \in N$ satisfying $\varepsilon_{j} \leq \frac{1}{n}$, by Fatou's Lemma, we have

$$
\int_{\Omega_{\frac{1}{n}}} \frac{\mathbf{m}^{2}}{\rho} d x \leq C .
$$

Now we fix $n \in N$ and denote the set of vacuum by $\mathcal{L}:=\left\{x \in \Omega_{\frac{1}{n}} \mid \rho(x, t)=0\right\}$. Note that $\sqrt{\rho^{j}} U^{j}$ converges almost everywhere to $\frac{\mathbf{m}}{\sqrt{\rho}}$ in the region $\mathcal{L}^{c}=\left\{x \in \Omega_{\frac{1}{n}} \mid \rho(x, t) \neq 0\right\}$. To control $\sqrt{\rho^{j}} U^{j}$ in the vacuum set, one sets $\mathcal{N}^{j}:=\left\{x \in \Omega_{\frac{1}{n}}\left|\left(\rho^{j}\right)^{\frac{1}{2+\alpha}}\right| U^{j} \mid \geq M\right\}$ for $M>0$ and small $\alpha>0$ to be specified later.

Consider

$$
\begin{aligned}
& \int_{0}^{T} \int_{\Omega_{\frac{1}{n}}}\left|\sqrt{\rho^{j}} U^{j}-\frac{\mathbf{m}}{\sqrt{\rho}}\right|^{2} d x d t \\
& \quad=\int_{0}^{T} \int_{\left(\mathcal{N}^{j}\right) \backslash \mathcal{L}}\left|\sqrt{\rho^{j}} U^{j}-\frac{\mathbf{m}}{\sqrt{\rho}}\right|^{2} d x d t+\int_{0}^{T} \int_{\left(\mathcal{N}^{j}\right) c \cap \mathcal{L}}\left|\sqrt{\rho^{j}} U^{j}-\frac{\mathbf{m}}{\sqrt{\rho}}\right|^{2} d x d t
\end{aligned}
$$




$$
\begin{aligned}
& +\int_{0}^{T} \int_{\mathcal{N}^{j}}\left|\sqrt{\rho^{j}} U^{j}-\frac{\mathbf{m}}{\sqrt{\rho}}\right|^{2} d x d t \\
=: & I_{1}+I_{2}+I_{3} .
\end{aligned}
$$

For the first term $I_{1}$ in (5.69), we note that in $\{\rho(x, t) \neq 0\}, \sqrt{\rho^{j}} U^{j}$ converges almost everywhere to $\frac{\mathbf{m}}{\sqrt{\rho}}$, and Lemma 5.6 shows that $\left\|\sqrt{\rho}^{j} U^{j}\right\|_{L^{\infty}\left(L^{2+2 \xi}\left(\Omega_{\frac{1}{n}}\right)\right)} \leq C$. By Egorov's theorem, we have

$$
\sqrt{\rho^{j}} U^{j} \rightarrow \frac{\mathbf{m}}{\sqrt{\rho}} \quad \text { strongly in } L^{2}\left(L^{2}\left(\Omega_{\frac{1}{n}}\right)\right),
$$

which means that $I_{1} \rightarrow 0$ as $j \rightarrow \infty$.

For the third term $I_{3}$, Tchebychev's inequality yields $\left|\mathcal{N}^{j}\right| \leq \frac{C}{M^{2}}$, and thus

$$
\begin{aligned}
I_{3} & =\int_{0}^{T} \int_{\mathcal{N}^{j}}\left|\sqrt{\rho^{j}} U^{j}-\frac{\mathbf{m}}{\sqrt{\rho}}\right|^{2} d x d t \\
& \leq \int_{0}^{T}\left(\int_{\mathcal{N}^{j}}\left|\sqrt{\rho^{j}} U^{j}-\frac{\mathbf{m}}{\sqrt{\rho}}\right|^{2+2 \xi} d x\right)^{\frac{1}{1+\xi}}\left(\int_{\mathcal{N}^{j}} d x\right)^{\frac{\xi}{1+\xi}} d t \\
& \leq C(T)\left(\left\|\sqrt{\rho}^{j} U^{j}\right\|_{L^{\infty}\left(L^{2+\xi}\left(\mathcal{N}^{j}\right)\right)}+\left\|\frac{\mathbf{m}}{\sqrt{\rho}}\right\|_{L^{\infty}\left(L^{2+\xi}\left(\mathcal{N}^{j}\right)\right)}\right)\left|\mathcal{N}^{j}\right|^{\frac{\xi}{1+\xi}} \\
& \leq \frac{C}{M^{\frac{2 \xi}{1+\xi}}} \rightarrow 0 \quad \text { as } M=M(j) \rightarrow \infty(\text { as } j \rightarrow \infty) .
\end{aligned}
$$

For the second term $I_{2}$, the definition of $\mathcal{N}^{j}$ implies that

$$
\sqrt{\rho^{j}} U^{j}=\left(\rho^{j}\right)^{\frac{1}{2+\alpha}} U^{j}\left(\rho^{j}\right)^{\frac{1}{2}-\frac{1}{2+\alpha}}<M\left(\rho^{j}\right)^{\frac{1}{2}-\frac{1}{2+\eta}} \rightarrow 0 \text { a.e., }
$$

when $\frac{1}{2}-\frac{1}{2+\alpha}>0$. That is, $\sqrt{\rho^{j}} U^{j} \rightarrow 0$ a.e. in $\left(\mathcal{N}^{j}\right)^{c} \cap \mathcal{L}$. Using Egorov's theorem yields

$$
\sqrt{\rho^{j}} U^{j} \rightarrow 0 \quad \text { strongly in } L^{2}\left(L^{2}\left(\left(\mathcal{N}^{j}\right)^{c} \cap \mathcal{L}\right)\right)
$$

which means

$$
I_{2}=\int_{0}^{T} \int_{\left(\mathcal{N}^{j}\right)^{c} \cap \mathcal{L}}\left|\sqrt{\rho^{j}} U^{j}\right|^{2} d x d t \rightarrow 0 \quad \text { as } j \rightarrow \infty .
$$

Combining all the arguments above and using the diagonal principle, we obtain that $\sqrt{\rho^{j}} U^{j}$ converges strongly in $L^{2}\left((0, T) \times \Omega_{\frac{1}{n}}\right)$ to $\frac{\mathbf{m}}{\sqrt{\rho}}$ for any positive integer $n$. The proof of this proposition is completed.

The following lemma can be directly obtained from Propositions 5.2 and 5.7; the details here are omitted.

Lemma 5.8 $\operatorname{Let}^{j}(r, t)=\rho^{j}(r, t) u^{j}(r, t)$. Then

(1) There exists a function $m(r, t)$ such that $\mathbf{m}(\mathbf{x}, t)=m(r, t) \frac{\mathbf{x}}{r}$ and $m^{j}(r, t)=\rho^{j}(r, t) u^{j}(r, t)$ converges to $m(r, t)$ strongly in $L^{2}\left(0, T ; L_{\mathrm{loc}}^{1+\beta}\left((0, R) ; r^{2} d r\right)\right)$. 
(2) There exists a function $u(r, t)$ such that $U(\mathbf{x}, t)=u(r, t) \frac{\mathbf{x}}{r}$ and the quantity $\sqrt{\rho^{j}} u^{j}$ converges strongly in $L^{2}\left(0, T ; L_{\text {loc }}^{2}\left((0, R) ; r^{2} d r\right)\right)$ to $\frac{m}{\sqrt{\rho}}$ (defined to be zero when $\left.m=0\right)$. Moreover, $m(r, t)=\rho u(r, t)$.

The following two propositions can be proved in a similar way as in [12], for the readers' convenience, we still give the detailed process.

Proposition 5.9 Let $(\rho, U)$ be the limit described as in Propositions 5.2, 5.3, 5.5 and 5.7, then (3.27) holds. Moreover, $\rho \in C\left([0, \infty) ; W^{1, \infty}(\Omega)^{*}\right)$, where $W^{1, \infty}(\Omega)^{*}$ is the dual space of $W^{1, \infty}(\Omega)$.

Proof Firstly, we derive the weak form of $(4.2)_{1}$. For any $\varphi(r, t) \in C^{1}\left([0, R] \times\left[t_{1}, t_{2}\right]\right)$, it follows from (4.2) that

$$
\left.\int_{\epsilon_{j}}^{R} \rho^{j} \varphi r^{2} d r\right|_{t_{1}} ^{t_{1}}-\int_{t_{1}}^{t_{1}} \int_{\epsilon_{j}}^{R}\left(\rho^{j} \varphi_{t}+\rho^{j} u^{j} \varphi_{r}\right) r^{2} d r d t=0
$$

then by (5.5) and (5.6), we obtain

$$
\begin{gathered}
\left.\int_{0}^{R} \rho^{j} \varphi r^{2} d r\right|_{t_{1}} ^{t_{1}}-\int_{t_{1}}^{t_{1}} \int_{0}^{R}\left(\rho^{j} \varphi_{t}+\rho^{j} u^{j} \varphi_{r}\right) r^{2} d r d t \\
=\left.\int_{0}^{\epsilon_{j}} \rho^{j} \varphi r^{2} d r\right|_{t_{1}} ^{t_{1}}-\int_{t_{1}}^{t_{1}} \int_{0}^{\epsilon_{j}} \rho^{j} \varphi_{t} r^{2} d r d t
\end{gathered}
$$

It can be easily obtained due to Proposition 5.2 that

$$
\begin{aligned}
& \left.\left.\int_{0}^{R} \rho^{j} \varphi r^{2} d r\right|_{t_{1}} ^{t_{1}} \rightarrow \int_{0}^{R} \rho \varphi r^{2} d r\right|_{t_{1}} ^{t_{1}} \\
& \int_{t_{1}}^{t_{1}} \int_{0}^{R} \rho^{j} \varphi_{t} r^{2} d r d t \rightarrow \int_{t_{1}}^{t_{1}} \int_{0}^{R} \rho \varphi_{t} r^{2} d r d t .
\end{aligned}
$$

Furthermore, (5.10) implies that $\sqrt{\rho^{j}}$ is bounded in $L^{\infty}\left(L^{q}(\Omega)\right), q \in[2,6]$, and Proposition 5.2 tells that $\sqrt{\rho^{j}} \rightarrow \sqrt{\rho}$ a.e., thus by Egorov's theorem, we have

$$
\sqrt{\rho^{j}} \rightarrow \sqrt{\rho} \text { strongly in } L^{2}\left(0, T ; L^{2}\left((0, R) ; r^{2} d r\right)\right)
$$

By a similar method, Lemma 5.6, part (1) of Proposition 5.7, and Egorov's theorem show that

$$
\sqrt{\rho^{j}} u^{j} \rightarrow \sqrt{\rho} u \quad \text { strongly in } L^{2}\left(0, T ; L^{2}\left((0, R) ; r^{2} d r\right)\right) .
$$

Equations (5.79) and (5.80) show that

$$
\int_{t_{1}}^{t_{1}} \int_{0}^{R} \rho^{j} u^{j} \varphi_{r} r^{2} d r d t \rightarrow \int_{t_{1}}^{t_{1}} \int_{0}^{R} \rho u \varphi_{r} r^{2} d r d t
$$


Now we prove that the right-hand side of (5.76) tends to zero as $j \rightarrow \infty$. Indeed,

$$
\int_{0}^{\epsilon_{j}} \rho^{j} \varphi r^{2} d r \leq C\left(\int_{0}^{\epsilon_{j}}\left(\rho^{j}\right)^{\frac{3}{2}} r^{2} d r\right)^{\frac{2}{3}}\left(\frac{\epsilon_{j}}{3}\right)^{\frac{1}{3}} \leq C \epsilon_{j}^{\frac{1}{3}} \rightarrow 0 \quad \text { as } j \rightarrow \infty .
$$

Similarly,

$$
\int_{t_{1}}^{t_{1}} \int_{0}^{\epsilon_{j}} \rho^{j} \varphi_{t} r^{2} d r d t \rightarrow 0 \quad \text { as } j \rightarrow \infty
$$

Therefore, we have proved that the weak form of $(3.5)_{1}$ is as follows:

$$
\left.\int_{0}^{R} \rho \varphi r^{2} d r\right|_{t_{1}} ^{t_{2}}=\int_{t_{1}}^{t_{2}} \int_{0}^{R}\left(\rho \varphi_{t}+\rho u \varphi_{r}\right) r^{2} d r d t
$$

Now let $\psi: \bar{\Omega} \times\left[t_{1}, t_{2}\right] \rightarrow R$ be any $C^{1}$ function. Define $\varphi(r, t):=\int_{S} \psi(r y, t) d S_{y}$, where the integral is over the unit sphere $S=S^{2}$ in $R^{3}$. Then

$$
\begin{aligned}
\int_{0}^{R} \rho(r, t) \varphi(r, t) r^{2} d r & =\int_{0}^{R} \int_{S} \rho(r, t) \psi(r y, t) r^{2} d S_{y} d r \\
& =\int_{\Omega} \rho(x, t) \psi(x, t) d x
\end{aligned}
$$

Similarly,

$$
\int_{t_{1}}^{t_{2}} \int_{0}^{R} \rho(r, t) \varphi_{t}(r, t) r^{2} d r d t=\int_{t_{1}}^{t_{2}} \int_{\Omega} \rho \psi_{t}(x, t) d x d t
$$

and

$$
\int_{t_{1}}^{t_{2}} \int_{0}^{R} \rho u \varphi_{r} r^{2} d r d t=\int_{t_{1}}^{t_{2}} \int_{\Omega} \rho(x, t) U(x, t) \cdot \nabla \psi(x, t) d x d t
$$

It follows from (5.85)-(5.87) that

$$
\left.\int_{\Omega} \rho \psi d x\right|_{t_{1}} ^{t_{2}}=\int_{t_{1}}^{t_{2}} \int_{\Omega}\left(\rho \psi_{t}+\rho U \cdot \nabla \psi\right) d x d t
$$

which establishes the weak form of the mass equation, i.e., (3.27) holds.

Now we prove that $\rho \in C\left([0, \infty) ; W^{1, \infty}(\Omega)^{*}\right)$. If $\zeta$ is a $C^{1}$ function of $x$, then by the continuity equation, we have

$$
\begin{aligned}
\left|\int_{\Omega} \rho \zeta d x\right|_{t_{1}}^{t_{2}} \mid & =\left|\int_{t_{1}}^{t_{2}} \int_{\Omega} \rho(x, t) U(x, t) \cdot \nabla \zeta(x, t) d x d t\right| \\
& \leq\|\nabla \zeta\|_{L^{\infty}} \int_{t_{1}}^{t_{2}}\left(\int_{\Omega} \rho d x\right)^{\frac{1}{2}}\left(\int_{\Omega} \rho U^{2} d x\right)^{\frac{1}{2}} d t \\
& \leq C(T)\|\nabla \zeta\|_{L^{\infty}\left|t_{2}-t_{1}\right| .}
\end{aligned}
$$


Gui and Song Boundary Value Problems

(2019) 2019:157

Page 31 of 40

By the density argument, we can extend this to functions $\zeta \in W^{1, \infty}(\Omega)$, so that

$$
\left\|\rho\left(\cdot, t_{2}\right)-\rho\left(\cdot, t_{1}\right)\right\|_{W^{1, \infty}(\Omega)^{*}}=\sup _{\|\zeta\|_{W^{1, \infty}=1}}\left|\int_{\Omega} \rho \tilde{\phi} d x\right|_{t_{1}}^{t_{2}}|\leq C(T)| t_{2}-t_{1} \mid
$$

for $t_{1}, t_{2} \in[0, T]$. The proof of this proposition is completed.

In the following, we prove that $(\rho, U)$ satisfies the weak form of the momentum equation $(3.1)_{2}$, and $(\rho, \Phi)$ satisfies the Poisson equation a.e., that is, (3.28) and (3.30) hold.

Proposition 5.10 Let $(\rho, U)$ be the limit described as in Propositions 5.2, 5.3, 5.5, and 5.7, then (3.28) and (3.30) hold.

Proof Let $\varphi$ be a $C^{2}$ function on $[0, R] \times[0, T]$ with $\varphi(0, t)=\varphi(R, t)=0$ for all $t \in[0, T]$, and $\varphi(r, T)=0$. Then using $(4.2)_{2}$ and integration by parts yields

$$
\begin{aligned}
\int_{0}^{T} & \int_{\epsilon_{j}}^{R}\left(\rho^{j} u^{j} \varphi_{t}+\rho^{j}\left|u^{j}\right|^{2} \varphi_{r}+\left(\rho^{j}\right)^{\gamma}\left(\varphi_{r}+\frac{2 \varphi}{r}\right)\right) r^{2} d r d t+\int_{\epsilon_{j}}^{R} m_{0}^{j}(r) \varphi(r, 0) r^{2} d r \\
& -\int_{0}^{T} \int_{\epsilon_{j}}^{R} \rho^{j}\left(u_{r}^{j} \varphi_{r}+\frac{2 u^{j} \varphi}{r^{2}}\right) r^{2} d r d t \\
= & \int_{0}^{T} \int_{\epsilon_{j}}^{R} \frac{3}{4} \epsilon_{j}\left(\rho^{j}\right)^{\frac{3}{4}}\left(u_{r}^{j}+\frac{2 u^{j}}{r}\right)\left(\varphi_{r}+\frac{2 \varphi}{r}\right) r^{2} d r d t-\int_{0}^{T} \int_{\epsilon_{j}}^{R} \epsilon_{j}\left(\rho^{j}\right)^{\frac{3}{4}}\left(\frac{2 u_{r}^{j} \varphi}{r}\right. \\
& \left.+\frac{2 u^{j} \varphi_{r}}{r}+\frac{2 u^{j} \varphi}{r^{2}}\right) r^{2} d r d t-\int_{0}^{T} \int_{\epsilon_{j}}^{R} \rho^{j} \phi_{r}^{j} \varphi r^{2} d r d t+\epsilon_{b}^{j},
\end{aligned}
$$

where

$$
\begin{aligned}
\epsilon_{b}^{j}= & \int_{0}^{T}\left\{\left(\rho^{j}\left(\epsilon_{j}, t\right)+\frac{3}{4} \epsilon_{j}\left(\rho^{j}\right)^{\frac{3}{4}}\left(\epsilon_{j}, t\right)\right) u_{r}^{j}\left(\epsilon_{j}, t\right) \varphi\left(\epsilon_{j}, t\right) \epsilon_{j}^{2}\right. \\
& \left.-\epsilon_{j}^{2}\left(\rho^{j}\right)^{\gamma}\left(\epsilon_{j}, t\right) \varphi\left(\epsilon_{j}, t\right)\right\} d t .
\end{aligned}
$$

We claim that

$$
\epsilon_{b}^{j} \rightarrow 0 \quad \text { as } \epsilon_{j} \rightarrow 0 .
$$

To check this, we first show that

$$
\lim _{\epsilon_{j} \rightarrow 0^{+}} \int_{0}^{T} \epsilon_{j}^{2}\left(\rho^{j}\right)^{\gamma}\left(\epsilon_{j}, t\right) \varphi\left(\epsilon_{j}, t\right) d t=0
$$

Indeed, note that

$$
\begin{aligned}
& \left|\epsilon_{j}^{2} \int_{0}^{T}\left(\rho^{j}\right)^{\gamma}\left(\epsilon_{j}, t\right) \varphi\left(\epsilon_{j}, t\right) d t\right| \\
& \quad \leq \max _{0 \leq t \leq T}\left|\varphi\left(\epsilon_{j}, t\right)\right|\left\{\int_{0}^{T} \int_{\epsilon_{j}}^{R}\left(\rho^{j}\right)^{\gamma}(r, t) r^{2} d r d t+\int_{0}^{T} \int_{\epsilon_{j}}^{R}\left|\partial_{r}\left(\rho^{j}\right)^{\gamma}(r, t)\right| r^{2} d r d t\right\}
\end{aligned}
$$




$$
\begin{aligned}
\leq & \max _{0 \leq t \leq T}\left|\varphi\left(\epsilon_{j}, t\right)\right|\left\{C+2\left(\int_{0}^{T} \int_{\epsilon_{j}}^{R}\left(\rho^{j}\right)^{\gamma}(r, t) r^{2} d r d t\right)^{\frac{1}{2}}\right. \\
& \left.\times\left(\int_{0}^{T} \int_{\epsilon_{j}}^{R}\left|\partial_{r}\left(\rho^{j}\right)^{\frac{\gamma}{2}}\right|^{2} r^{2} d r d t\right)^{\frac{1}{2}}\right\} \\
\leq & \max _{0 \leq t \leq T}\left|\varphi\left(\epsilon_{j}, t\right)\right|\left\{C+\int_{0}^{T} \int_{\epsilon_{j}}^{R}\left(\rho^{j}\right)^{\gamma}(r, t) r^{2} d r d t+\int_{0}^{T} \int_{\epsilon_{j}}^{R}\left|\partial_{r}\left(\rho^{j}\right)^{\frac{\gamma}{2}}\right|^{2} r^{2} d r d t\right\} \\
\leq & C \max _{0 \leq t \leq T}\left|\varphi\left(\epsilon_{j}, t\right)\right| \rightarrow 0 \text { as } \epsilon_{j} \rightarrow 0,
\end{aligned}
$$

i.e., (5.94) holds.

Next we show that

$$
\lim _{\epsilon_{j} \rightarrow 0^{+}} \int_{0}^{T}\left(\rho^{j} u_{r}^{j}\right)\left(\epsilon_{j}, t\right) \varphi\left(\epsilon_{j}, t\right) \epsilon_{j}^{2} d t=0 .
$$

Thanks to (4.2) ${ }_{1}$ and the boundary condition $u\left(\epsilon_{j}, t\right)=0$, one has

$$
\rho_{t}^{j}\left(\epsilon_{j}, t\right)+\rho^{j}\left(\epsilon_{j}, t\right) u_{r}^{j}\left(\epsilon_{j}, t\right)=0 .
$$

Thus, by integration by parts, we have

$$
\begin{aligned}
& \lim _{\epsilon_{j} \rightarrow 0^{+}} \int_{0}^{T}\left(\rho^{j} u_{r}^{j}\right)\left(\epsilon_{j}, t\right) \varphi\left(\epsilon_{j}, t\right) \epsilon_{j}^{2} d t \\
& \quad=\lim _{\epsilon_{j} \rightarrow 0^{+}}\left\{-\epsilon_{j}^{2} \int_{0}^{T} \rho_{t}^{j}\left(\epsilon_{j}, t\right) \varphi\left(\epsilon_{j}, t\right) d t\right\} \\
& \quad=\lim _{\epsilon_{j} \rightarrow 0^{+}}\left\{\epsilon_{j}^{2} \int_{0}^{T} \rho^{j}\left(\epsilon_{j}, t\right) \varphi_{t}\left(\epsilon_{j}, t\right) d t+\epsilon_{j}^{2}\left(\rho_{0}\left(\epsilon_{j}\right)+\epsilon_{j}\right) \varphi\left(\epsilon_{j}, 0\right)\right\} \\
& \quad=\lim _{\epsilon_{j} \rightarrow 0^{+}} \epsilon_{j}^{2} \int_{0}^{T} \rho^{j}\left(\epsilon_{j}, t\right) \varphi_{t}\left(\epsilon_{j}, t\right) d t .
\end{aligned}
$$

On the other hand, it is easy to get

$$
\begin{aligned}
\epsilon_{j}^{2}\left|\int_{0}^{T} \rho^{j}\left(\epsilon_{j}, t\right) \varphi_{t}\left(\epsilon_{j}, t\right) d t\right| & \leq \epsilon_{j}^{2-\frac{2}{\gamma}}\left\{\epsilon_{j}^{2} \int_{0}^{T}\left(\rho^{j}\right)^{\gamma}\left(\epsilon_{j}, t\right) d t\right\}^{\frac{1}{\gamma}}\left\{\int_{0}^{T} \varphi_{t}^{\frac{\gamma}{\gamma-1}}(\epsilon, t) d t\right\}^{\frac{\gamma-1}{\gamma}} \\
& \leq C \epsilon_{j}^{2-\frac{2}{\gamma}} \rightarrow 0 \text { as } \epsilon_{j} \rightarrow 0^{+}(1<\gamma<3),
\end{aligned}
$$

thus, (5.96) holds.

Similarly, one can show that

$$
\lim _{\epsilon_{j} \rightarrow 0^{+}} \frac{3}{4} \epsilon_{j} \int_{0}^{T} \epsilon_{j}^{2}\left\{\left(\rho^{j}\right)^{\frac{3}{4}} u_{r}^{j}\right\}\left(\epsilon_{j}, t\right) \varphi\left(\epsilon_{j}, t\right) d t=0 .
$$

Indeed, using (5.97) and $\rho>0$ yields

$$
\left(\rho^{j}\right)^{-\frac{1}{4}} \rho_{t}^{j}\left(\epsilon_{j}, t\right)+\left(\rho^{j}\right)^{\frac{3}{4}}\left(\epsilon_{j}, t\right) u_{r}^{j}\left(\epsilon_{j}, t\right)=0,
$$


i.e.,

$$
\frac{4}{3} \partial_{t}\left(\rho^{j}\right)^{\frac{3}{4}}\left(\epsilon_{j}, t\right)+\left(\rho^{j}\right)^{\frac{3}{4}}\left(\epsilon_{j}, t\right) u_{r}^{j}\left(\epsilon_{j}, t\right)=0 .
$$

Integrating by parts, we have

$$
\begin{aligned}
& \frac{3}{4} \epsilon_{j} \int_{0}^{T} \epsilon_{j}^{2}\left\{\left(\rho^{j}\right)^{\frac{3}{4}} u_{r}^{j}\right\}\left(\epsilon_{j}, t\right) \varphi\left(\epsilon_{j}, t\right) d t \\
& \quad=-\int_{0}^{T} \epsilon_{j}^{3} \partial_{t}\left(\rho^{j}\right)^{\frac{3}{4}}\left(\epsilon_{j}, t\right) \varphi\left(\epsilon_{j}, t\right) d t \\
& \quad=\int_{0}^{T} \epsilon_{j}^{3}\left(\rho^{j}\right)^{\frac{3}{4}}\left(\epsilon_{j}, t\right) \varphi_{t}\left(\epsilon_{j}, t\right) d t+\epsilon_{j}^{3}\left(\rho_{0}\left(\epsilon_{j}\right)+\epsilon_{j}\right)^{\frac{3}{4}} \varphi_{t}\left(\epsilon_{j}, 0\right) \\
& \quad \leq \epsilon_{j}^{3-\frac{3}{2 \gamma}}\left\{\epsilon_{j}^{2} \int_{0}^{T}\left(\rho^{j}\right)^{\gamma}\left(\epsilon_{j}, t\right) d t\right\}^{\frac{3}{4 \gamma}}\left\{\int_{0}^{T} \varphi_{t}^{\frac{4 \gamma}{4 \gamma-3}}\left(\epsilon_{j}, t\right) d t\right\}^{\frac{4 \gamma-3}{4 \gamma}}+\epsilon_{j}^{3}\left(\rho_{0}+\epsilon_{j}\right)^{\frac{3}{4}} \varphi_{t}\left(\epsilon_{j}, 0\right) \\
& \quad \leq C \epsilon_{j}^{3-\frac{3}{2 \gamma}}+\epsilon_{j}^{3}\left(\rho_{0}+\epsilon_{j}\right)^{\frac{3}{4}} \varphi_{t}\left(\epsilon_{j}, 0\right) \rightarrow 0 \text { as } \epsilon_{j} \rightarrow 0(1<\gamma<3),
\end{aligned}
$$

i.e., (5.100) holds. Equation (5.93) is a consequence of (5.94), (5.96), and (5.100).

Now, for any $\omega=\left(\omega^{1}, \omega^{2}, \omega^{3}\right) \in C^{2}(\bar{\Omega} \times[0, T])$ satisfying $\omega(x, t)=0$ on $\partial \Omega$ and $\omega(x, T)=$ 0 , set $\varphi(r, t):=\int_{S} w(r y, t) \cdot y d S_{y}$, where $S=S^{2}$ is the unit sphere in $R^{3}$, and note that

$$
\begin{aligned}
\left(r^{2} \varphi\right)_{r} & =\partial_{r} \int_{S_{y}} w(r y, t) \cdot y r^{2} d S_{y} \\
& =\partial_{r} \int_{|x| \leq r} \operatorname{div} w(x, t) d x \\
& =\int_{|x|=r} \operatorname{div} w(x, t) d S_{x} \\
& =r^{2} \int_{S_{y}}\left(w^{i}\right)_{x_{i}}(r y, t) d S_{y} .
\end{aligned}
$$

Now we convert every term in (5.91) to the form in Cartesian coordinates.

Direct calculations show that

$$
\int_{\epsilon_{j}}^{R} m_{0}^{j}(r) \varphi(r, 0) r^{2} d r=\int_{\Omega_{\epsilon_{j}}} \mathbf{m}_{0}^{j}(x) \cdot w(x, 0) d x
$$

Similarly, $\int_{0}^{T} \int_{\epsilon_{j}}^{R} \rho^{j} u^{j} \varphi_{t} r^{2} d r d t=\int_{0}^{T} \int_{\Omega_{\epsilon_{j}}} \sqrt{\rho^{j}}\left(\sqrt{\rho^{j}} U^{j}\right) \cdot \partial_{t} w d x d t$,

$$
\begin{aligned}
\int_{0}^{T} \int_{\epsilon_{j}}^{R} \rho^{j}\left(u^{j}\right)^{2} \varphi_{r} r^{2} d r d t & =\int_{0}^{T} \int_{\epsilon_{j}}^{R} \int_{|x|=r} \rho^{j}\left(u^{j}\right)^{2} \frac{x_{i}}{r} \frac{x_{k}}{r}\left(w^{i}\right)_{x_{k}}(x, t) d S_{x} d r d t \\
& =\int_{0}^{T} \int_{\Omega_{\epsilon_{j}}} \sqrt{\rho^{j}} U^{j} \otimes \sqrt{\rho^{j}} U^{j}: \nabla w d x d t
\end{aligned}
$$


Gui and Song Boundary Value Problems

(2019) 2019:157

Page 34 of 40

and

$$
\begin{aligned}
\int_{0}^{T} \int_{\epsilon_{j}}^{R}\left(\rho^{j}\right)^{\gamma}\left(\varphi_{r}+\frac{2 \varphi}{r}\right) r^{2} d r d t & =\int_{0}^{T} \int_{\epsilon_{j}}^{R}\left(\rho^{j}\right)^{\gamma}\left(r^{2} \varphi\right)_{r} d r d t \\
& =\int_{0}^{T} \int_{\epsilon_{j}}^{R} \int_{|x|=r} \operatorname{div} w(x, t)\left(\rho^{j}\right)^{\gamma} d S_{x} d r d t \\
& =\int_{0}^{T} \int_{\Omega_{\epsilon_{j}}}\left(\rho^{j}\right)^{\gamma} \operatorname{div} w(x, t) d x d t
\end{aligned}
$$

due to (5.104).

Moreover,

$$
\begin{aligned}
& -\int_{0}^{T} \int_{\epsilon_{j}}^{R} \rho^{j}\left(u_{r}^{j} \varphi_{r}+\frac{2 u^{j} \varphi}{r^{2}}\right) r^{2} d r d t \\
& =-\int_{0}^{T} \int_{\epsilon_{j}}^{R} \rho^{j}\left\{\left(\frac{u^{j}}{r}\right)_{r} r \varphi_{r}+\frac{u^{j}}{r} r^{-2}\left(r^{2} \varphi\right)_{r}\right\} r^{2} d r d t \\
& =-\int_{0}^{T} \int_{\epsilon_{j}}^{R} \rho^{j}\left\{\left(\frac{u^{j}}{r}\right)_{r} r \int_{S}\left(w^{i}\right)_{x_{k}}(r y, t) y_{i} y_{k} d S_{y}+\frac{u^{j}}{r} \int_{S} \operatorname{div} w(r y, t) d S_{y}\right\} r^{2} d r d t \\
& =-\int_{0}^{T} \int_{\Omega_{\epsilon_{j}}} \rho^{j}\left\{\left(\frac{u^{j}}{r}\right)_{r} \frac{x_{i} x_{k}}{r}+\frac{u^{j}}{r} \delta_{i k}\right\}\left(w^{i}\right)_{x_{k}} d x d t \\
& =-\int_{0}^{T} \int_{\Omega_{\epsilon_{j}}} \rho^{j} \frac{\partial}{\partial x_{k}}\left(u^{j} \frac{x_{i}}{r}\right)\left(w^{i}\right)_{x_{k}} d x d t \\
& =-\int_{0}^{T} \int_{\Omega_{\epsilon_{j}}} \rho^{j} \nabla U^{j}: \nabla w d x d t, \\
& \int_{0}^{T} \int_{\epsilon_{j}}^{R} \frac{3}{4} \epsilon_{j}\left(\rho^{j}\right)^{\frac{3}{4}}\left(u_{r}^{j}+\frac{2 u^{j}}{r}\right)\left(\varphi_{r}+\frac{2 \varphi}{r}\right) r^{2} d r d t \\
& =\int_{0}^{T} \int_{\epsilon_{j}}^{R} \int_{|x|=r} \frac{3}{4} \epsilon_{j}\left(\rho^{j}\right)^{\frac{3}{4}} \operatorname{div} U^{j} \operatorname{div} w^{j}(x, t) d S_{x} d r d t \\
& =\int_{0}^{T} \int_{\Omega_{\epsilon_{j}}} \frac{3}{4} \epsilon_{j}\left(\rho^{j}\right)^{\frac{3}{4}} \operatorname{div} U^{j} \operatorname{div} w^{j} d x d t, \\
& -\int_{0}^{T} \int_{\epsilon_{j}}^{R} \epsilon_{j}\left(\rho^{j}\right)^{\frac{3}{4}}\left(\frac{2 u_{r}^{j} \varphi}{r}+\frac{2 u^{j} \varphi_{r}}{r}+\frac{2 u^{j} \varphi}{r^{2}}\right) r^{2} d r d t \\
& =-\int_{0}^{T} \int_{\Omega_{\epsilon_{j}}} \epsilon_{j}\left(\rho^{j}\right)^{\frac{3}{4}} \nabla U^{j}: \nabla w d x d t,
\end{aligned}
$$

and

$$
\begin{aligned}
-\int_{0}^{T} \int_{\epsilon_{j}}^{R} \rho^{j} \phi_{r}^{j} \varphi r^{2} d r d t & =-\int_{0}^{T} \int_{\epsilon_{j}}^{R} \rho^{j} \phi_{r}^{j} \int_{|x|=r} w(x, t) \cdot \frac{x}{r} r^{2} d S_{x} r^{2} d r d t \\
& =-\int_{0}^{T} \int_{\Omega_{\epsilon_{j}}} \rho^{j} \phi_{r}^{j} w(x, t) \cdot \frac{x}{r} d x d t \\
& =-\int_{0}^{T} \int_{\Omega_{\epsilon_{j}}} \rho^{j} \nabla \Phi^{j} w(x, t) d x d t
\end{aligned}
$$


Thus, it follows from (5.91) that

$$
\begin{aligned}
\int_{\Omega_{\epsilon_{j}}} & \mathbf{m}_{0}^{j}(x) \cdot w(x, 0) d x+\int_{0}^{T} \int_{\Omega_{\epsilon_{j}}}\left\{\sqrt{\rho^{j}}\left(\sqrt{\rho^{j}} U^{j}\right) \cdot \partial_{t} w+\sqrt{\rho^{j}} U^{j} \otimes \sqrt{\rho^{j}} U^{j}: \nabla w\right\} d x d t \\
& +\int_{0}^{T} \int_{\Omega_{\epsilon_{j}}}\left(\rho^{j}\right)^{\gamma} \operatorname{div} w d x d t-\int_{0}^{T} \int_{\Omega_{\epsilon_{j}}} \rho^{j} \nabla U^{j}: \nabla w d x d t \\
= & \int_{0}^{T} \int_{\Omega_{\epsilon_{j}}} \frac{3}{4} \epsilon_{j}\left(\rho^{j}\right)^{\frac{3}{4}} \operatorname{div} U^{j} \operatorname{div} w^{j} d x d t-\int_{0}^{T} \int_{\Omega_{\epsilon_{j}}} \epsilon_{j}\left(\rho^{j}\right)^{\frac{3}{4}} \nabla U^{j}: \nabla w d x d t \\
& -\int_{0}^{T} \int_{\Omega_{\epsilon_{j}}} \rho^{j} \nabla \Phi^{j} \cdot w d x d t+\epsilon_{b^{j}} .
\end{aligned}
$$

Thanks to (5.5), one has

$$
\begin{aligned}
\int_{\Omega} \mathbf{m}_{0}^{j}(x) \cdot w(x, 0) d x+\int_{0}^{T} \int_{\Omega}\left(\sqrt{\rho^{j}}\left(\sqrt{\rho^{j}} U^{j}\right) w_{t}+\sqrt{\rho^{j}} U^{j} \otimes \sqrt{\rho^{j}} U^{j}: \nabla w\right) d x d t \\
\quad+\int_{0}^{T} \int_{\Omega}\left(\rho^{j}\right)^{\gamma} \operatorname{div} w(x, t) d x d t-\int_{0}^{T} \int_{\Omega} \rho^{j} \nabla U^{j}: \nabla w d x d t \\
\quad+\int_{0}^{T} \int_{\Omega} \rho^{j} \nabla \phi^{j} w(x, t) d x d t \\
=\int_{0}^{T} \int_{B_{\epsilon_{j}}}\left(\rho^{j}\right)^{\gamma} \operatorname{div} w d x d t+\int_{0}^{T} \int_{\Omega_{\epsilon_{j}}} \frac{3}{4} \epsilon_{j}\left(\rho^{j}\right)^{\frac{3}{4}} \operatorname{div} U^{j} \operatorname{div} w^{j} d x d t \\
\quad-\int_{0}^{T} \int_{\Omega_{\epsilon_{j}}} \epsilon_{j}\left(\rho^{j}\right)^{\frac{3}{4}} \nabla U^{j}: \nabla w d x d t+\int_{0}^{T} \int_{B_{\epsilon_{j}}} \rho^{j} \nabla \Phi^{j} \cdot w d x d t+\epsilon_{b^{j} .}
\end{aligned}
$$

We now prove that each term on the left-hand side of (5.113) converges to a corresponding term in (3.28) and each term on the right-hand side of (5.113) vanishes as $j \rightarrow 0$.

Obviously,

$$
\int_{\Omega} \mathbf{m}_{0}^{j}(x) \cdot w(x, 0) d x \rightarrow \int_{\Omega} \mathbf{m}_{0} \cdot w(x, 0) d x
$$

and, similar to the process of the proof in Proposition 5.9, it holds that

$$
\int_{0}^{T} \int_{\Omega} \sqrt{\rho^{j}}\left(\sqrt{\rho^{j}} U^{j}\right) \cdot \partial_{t} w d x d t \rightarrow \int_{0}^{T} \int_{\Omega} \sqrt{\rho}(\sqrt{\rho} U) \cdot \partial_{t} w d x d t
$$

Moreover,

$$
\begin{aligned}
\int_{0}^{T} & \int_{\Omega}\left(\sqrt{\rho^{j}} U^{j} \otimes \sqrt{\rho^{j}} U^{j}-\sqrt{\rho} U \otimes \sqrt{\rho} U\right): \nabla w d x d t \\
= & \int_{0}^{T} \int_{B_{\frac{1}{n}}}\left(\sqrt{\rho^{j}} U^{j} \otimes \sqrt{\rho^{j}} U^{j}-\sqrt{\rho} U \otimes \sqrt{\rho} U\right): \nabla w d x d t \\
& +\int_{0}^{T} \int_{\Omega_{\frac{1}{n}}}\left(\sqrt{\rho^{j}} U^{j} \otimes \sqrt{\rho^{j}} U^{j}-\sqrt{\rho} U \otimes \sqrt{\rho} U\right): \nabla w d x d t
\end{aligned}
$$




$$
\begin{aligned}
\leq & \|\nabla w\|_{L^{\infty}\left(B_{\frac{1}{n}}\right)} \int_{0}^{T} \int_{B_{\frac{1}{n}}}\left|\sqrt{\rho^{j}} U^{j}\right|^{2}+|\sqrt{\rho} U|^{2} d x d t \\
& +\left|\int_{0}^{T} \int_{\Omega_{\frac{1}{n}}}\left(\sqrt{\rho^{j}} U^{j} \otimes \sqrt{\rho^{j}} U^{j}-\sqrt{\rho} U \otimes \sqrt{\rho} U\right): \nabla w d x d t\right|,
\end{aligned}
$$

for any positive integer $n$. By virtue of (5.5) and Proposition 5.5, one has

$$
\begin{aligned}
\int_{0}^{T} \int_{B_{\frac{1}{n}}}\left|\sqrt{\rho^{j}} U^{j}\right|^{2} d x d t & \leq\left(\int_{0}^{T} \int_{B_{\frac{1}{n}}} \rho^{j} d x d t\right)^{\frac{\eta}{2+\eta}}\left(\int_{0}^{T} \int_{B_{\frac{1}{n}}} \rho^{j}\left|U^{j}\right|^{2+\eta} d x d t\right)^{\frac{2}{2+\eta}} \\
& \leq C\left(\int_{0}^{T} \int_{B_{\frac{1}{n}}} \rho^{j} d x d t\right)^{\frac{\eta}{2+\eta}} \\
& \leq C\left\{\left(\int_{0}^{T} \int_{B_{\frac{1}{n}}}\left(\rho^{j}\right)^{3} d x d t\right)^{\frac{1}{3}}\left|B_{\frac{1}{n}}\right|^{\frac{2}{3}}\right\}^{\frac{\eta}{2+\eta}} \\
& \leq C\left|B_{\frac{1}{n}}\right|^{\frac{2}{3} \cdot \frac{\eta}{2+\eta}} \rightarrow 0 \text { as } n \rightarrow \infty
\end{aligned}
$$

i.e.,

$$
\int_{0}^{T} \int_{B_{\frac{1}{n}}}\left|\sqrt{\rho^{j}} U^{j}\right|^{2} d x d t \rightarrow 0
$$

uniformly in $j$ as $n \rightarrow \infty$. Also,

$$
\int_{0}^{T} \int_{B_{\frac{1}{n}}}|\sqrt{\rho} U|^{2} d x d t \leq \liminf _{j \rightarrow \infty} \int_{0}^{T} \int_{B_{\frac{1}{n}}}\left|\sqrt{\rho^{j}} U^{j}\right|^{2} d x d t \rightarrow 0,
$$

as $n \rightarrow \infty$. It follows from (5.119) and Proposition 5.7 that

$$
\int_{0}^{T} \int_{\Omega} \sqrt{\rho^{j}} U^{j} \otimes \sqrt{\rho^{j}} U^{j}: \nabla w d x d t \rightarrow \int_{0}^{T} \int_{\Omega} \sqrt{\rho} U \otimes \sqrt{\rho} U: \nabla w d x d t
$$

as $j \rightarrow \infty$.

For the pressure term, Proposition 5.3 implies that

$$
\int_{0}^{T} \int_{\Omega}\left(\rho^{j}\right)^{\gamma} \operatorname{div} w d x d t \rightarrow \int_{0}^{T} \int_{\Omega} \rho^{\gamma} \operatorname{div} w d x d t, \quad j \rightarrow \infty .
$$

Concerning the diffusion terms on the left-hand side of (5.113), it follows from (5.5) and integration by parts that

$$
\begin{aligned}
\int_{0}^{T} & \int_{\Omega} \rho^{j} \nabla U^{j}: \nabla w d x d t \\
= & -\int_{0}^{T} \int_{\Omega} \sqrt{\rho^{j}}\left(\sqrt{\rho^{j}} U^{j}\right) \cdot \Delta w d x d t \\
& -2 \int_{0}^{T} \int_{\Omega}\left(\sqrt{\rho^{j}} U^{j}\right) \cdot\left(\nabla \sqrt{\rho^{j}} \cdot \nabla\right) w d x d t .
\end{aligned}
$$


Similar to the proof of (5.115), one can prove the convergence for the first term on the right-hand side of (5.122) as follows:

$$
\int_{0}^{T} \int_{\Omega} \sqrt{\rho^{j}}\left(\sqrt{\rho^{j}} U^{j}\right) \cdot \Delta w d x d t \rightarrow \int_{0}^{T} \int_{\Omega} \sqrt{\rho}(\sqrt{\rho} U) \cdot \Delta w d x d t, \quad j \rightarrow \infty .
$$

Due to Lemma 5.1, it holds that

$$
\left\|\nabla \sqrt{\rho^{j}}\right\|_{L^{\infty}\left(L^{2}(\Omega)\right)} \leq C
$$

and hence there exists a function $g \in L^{\infty}\left(L^{2}(\Omega)\right)$ such that

$$
\nabla \sqrt{\rho^{j}} \rightarrow g \text { weakly in } L^{2}\left(L^{2}(\Omega)\right)
$$

Lemma 5.1 and Proposition 5.2 imply that $\sqrt{\rho^{j}} \rightarrow \sqrt{\rho}$ weakly in $L^{2}\left(L^{2}(\Omega)\right)$, hence, $g=$ $\nabla \sqrt{\rho}$. Consequently, this yields that

$$
\nabla \sqrt{\rho^{j}} \rightarrow \nabla \sqrt{\rho} \text { weakly in } L^{2}\left(L^{2}(\Omega)\right)
$$

By Propositions 5.5 and 5.7, we obtain

$$
\begin{aligned}
& -2 \int_{0}^{T} \int_{\Omega}\left(\sqrt{\rho^{j}} U^{j}\right) \cdot\left(\nabla \sqrt{\rho^{j}} \cdot \nabla\right) w d x d t \\
& \rightarrow-2 \int_{0}^{T} \int_{\Omega}(\sqrt{\rho} U) \cdot(\nabla \sqrt{\rho} \cdot \nabla) w d x d t .
\end{aligned}
$$

Substituting (5.123) and (5.127) into (5.122) yields

$$
\begin{aligned}
& \int_{0}^{T} \int_{\Omega} \rho^{j} \nabla U^{j}: \nabla w d x d t \rightarrow\langle\rho \nabla U, \nabla \psi\rangle \\
& \quad=-\int_{0}^{T} \int_{\Omega} \sqrt{\rho}(\sqrt{\rho} U) \cdot \Delta \psi d x d t-2 \int_{0}^{T} \int_{\Omega}(\sqrt{\rho} U) \cdot(\nabla \sqrt{\rho} \cdot \nabla) \psi d x d t .
\end{aligned}
$$

Now, thanks to (5.5), (5.45) and Proposition 5.2, we have

$$
\int_{0}^{T} \int_{\Omega} \rho^{j} \nabla \Phi^{j} \cdot w d x d t \rightarrow \int_{0}^{T} \int_{\Omega} \rho \nabla \Phi \cdot w d x d t
$$

Up to now, we have proved that the terms on the left-hand side of (5.113) converge to corresponding ones in (3.28) as $j \rightarrow \infty$. In the following, we prove that each term on the right-hand side of (5.113) vanishes as $j \rightarrow \infty$.

Applying (5.5), (5.45) and Lemma 5.1, one can get

$$
\int_{0}^{T} \int_{\Omega}\left|\rho^{j} \nabla \Phi^{j}\right|^{2} d x d t \leq\left\{\int_{0}^{T} \int_{\Omega}\left(\rho^{j}\right)^{3} d x d t\right\}^{\frac{2}{3}}\left\{\int_{0}^{T} \int_{\Omega}\left|\nabla \Phi^{j}\right|^{6} d x d t\right\}^{\frac{1}{3}} \leq C,
$$


thus,

$$
\begin{aligned}
\int_{0}^{T} \int_{B_{\epsilon_{j}}} \rho^{j} \nabla \Phi^{j} \cdot w d x d t & \leq C(T)\left(\int_{0}^{T} \int_{\Omega}\left|\rho^{j} \nabla \Phi^{j}\right|^{2} d x d t\right)^{\frac{1}{2}}\left|B_{\epsilon_{j}}\right|^{\frac{1}{2}} \\
& \leq C(T)\left|B_{\epsilon_{j}}\right|^{\frac{1}{2}} \rightarrow 0 \text { as } j \rightarrow \infty, \\
\left|\int_{0}^{T} \int_{B_{\epsilon_{j}}}\left(\rho^{j}\right)^{\gamma} \operatorname{div} w d x d t\right| & \leq C\left(\int_{0}^{T} \int_{B_{\epsilon_{j}}}\left(\rho^{j}\right)^{3} d x d t\right)^{\frac{\gamma}{3}}\left|B_{\epsilon_{j}}\right|^{\frac{3-\gamma}{3}} \\
& \leq C\left|B_{\epsilon_{j}}\right|^{\frac{3-\gamma}{3}} \rightarrow 0,
\end{aligned}
$$

as $j \rightarrow \infty$, for $1<\gamma<3$.

Next, with the help of Lemma 5.1 again, one has

$$
\begin{aligned}
& \left|\int_{0}^{T} \int_{\Omega_{\epsilon_{j}}} \frac{3}{4} \epsilon_{j}\left(\rho^{j}\right)^{\frac{3}{4}} \operatorname{div} U^{j} \operatorname{div} w^{j} d x d t\right| \\
& \quad \leq C \sqrt{\epsilon_{j}}\left\{\epsilon_{j} \int_{0}^{T} \int_{\Omega_{\epsilon_{j}}}\left(\rho^{j}\right)^{\frac{3}{4}}\left|\nabla U^{j}\right|^{2} d x d t\right\}^{\frac{1}{2}}\left\{\int_{0}^{T} \int_{\Omega_{\epsilon_{j}}}\left(\rho^{j}\right)^{\frac{3}{4}} d x d t\right\}^{\frac{1}{2}} \\
& \quad \leq C \sqrt{\epsilon_{j}} \rightarrow 0 \text { as } j \rightarrow \infty .
\end{aligned}
$$

Similarly, the integral $\epsilon_{j} \int_{0}^{T} \int_{\Omega_{\epsilon_{j}}}\left(\rho^{j}\right)^{\frac{3}{4}} \nabla U^{j}: \nabla w d x d t$ admits the same bound as in (5.133). It follows from the above arguments that each term on the right-hand side of (5.113) converges to zero as $j \rightarrow \infty$. Equation (3.28) can be easily obtained by taking the limit $j \rightarrow \infty$ in (5.113). Furthermore, (3.30) can be easily obtained by using (5.5), (5.45) and Proposition 5.2. The proof of this proposition is completed.

Now we are ready to prove Theorem 3.4.

Proof of Theorem 3.4 The weak forms of the mass and momentum equations follow from Propositions 5.9 and 5.10, and Proposition 5.10 also shows that Poisson equation (3.1) holds almost everywhere. The first part in Definition 3.3 follows from Lemmas 4.2, 5.1 and Proposition 5.9. Moreover, (3.33)-(3.36) are obtained from Lemma 5.1 and Propositions 5.2, 5.9. Finally, the radial symmetry of the weak solutions is a consequence of Lemma 5.8. The proof of Theorem 3.4 is thus finished.

\section{Acknowledgements}

The authors are thankful to the learned reviewers for their time and efforts in reviewing our article. The quality of the article has been highly enhanced via the help of their valuable and constructive comments/suggestions. Also, the authors would like to thank Prof. Zhenhua Guo for the discussions and support.

\section{Funding}

National Natural Science Foundation of China, No. 11801443. The research project of science and technology bureau of Yulin city, No. 2014cxy04-01.

Availability of data and materials

Not applicable. 
Authors' contributions

Both authors have made equal contributions to the publication of this article. Both authors read and approved the final manuscript.

\section{Publisher's Note}

Springer Nature remains neutral with regard to jurisdictional claims in published maps and institutional affiliations.

Received: 2 March 2019 Accepted: 24 September 2019 Published online: 01 October 2019

\section{References}

1. Bresch, D., Desjardins, B.: Existence of global weak solutions for a $2 \mathrm{D}$ viscous shallow water equations and convergence to the quasi-geostrophic model. Commun. Math. Phys. 238, 211-223 (2003)

2. Bresch, D., Desjardins, B., Gerard-Varet, D.: On compressible Navier-Stokes equations with density dependent viscosities in bounded domains. J. Math. Pures Appl. 87, 227-235 (2007)

3. Bresch, D., Desjardins, B., Lin, C.K.: On some compressible fluid models: Korteweg, lubrication, and shallow water systems. Commun. Partial Differ. Equ. 28(3-4), 843-868 (2003)

4. Cai, H., Tan, Z:: Weak time-periodic solutions to the compressible Navier-Stokes-Poisson equations. Commun. Math. Sci. 13, 1515-1540 (2015)

5. Documet, B., Feireisl, E.: On the dynamics of gaseous stars. Arch. Ration. Mech. Anal. 174, 221-266 (2004)

6. Documet, B., Feireisl, E., Petzeltova, H., Straskraba, l.: Global in time weak solutions for compressible barotropic self-gravitating fluids. Discrete Contin. Dyn. Syst. 11, 113-130 (2004)

7. Donatelli, D.: Local and global existence for the coupled Navier-Stokes-Poisson problem. Q. Appl. Math. 61, 345-361 (2003)

8. Duan, Q.: On the dynamics of Navier-Stokes equations for a spherically symmetric shallow water model. J. Math. Anal. Appl. 404, 260-282 (2013)

9. Duan, Q., Li, H.L.: Global existence of weak solution for the compressible Navier-Stokes-Poisson system for gaseous stars. J. Differ. Equ. 259, 5302-5330 (2015)

10. Ducomet, B., Nečasová, Š., Vasseur, A.: On spherically symmetric motions of a viscous compressible barotropic and selfgravitating gas. J. Math. Fluid Mech. 13, 191-211 (2011)

11. Feireisl, E., Novotny, A., Petzeltova, H.: On the existence of globally defined weak solutions to the Navier-Stokes equations. J. Math. Fluid Mech. 3, 358-392 (2001)

12. Guo, Z.H., Jiu, Q.S., Xin, Z.P.: Spherically symmetric isentropic compressible flows with density-dependent viscosity coefficients. SIAM J. Math. Anal. 39, 1402-1427 (2008)

13. Guo, Z.H., Li, H.L., Xin, Z.P.: Lagrange structure and dynamics for spherically symmetric compressible Navier-Stokes equations. Commun. Math. Phys. 309(2), 371-412 (2012)

14. Hoff, D.: Global existence for 1D, compressible, isentropic Navier-Stokes equations with large initial data. Trans. Am. Math. Soc. 303, 169-181 (1987)

15. Hoff, D., Santos, M.: Lagrangian structure and propagation of singularities in multidimensional compressible flow. Arch. Ration. Mech. Anal. 188(3), 509-543 (2008)

16. Hoff, D., Serre, D.: The failure of continuous dependence on initial data for the Navier-Stokes equations of compressible flow. SIAM J. Appl. Math. 51, 887-898 (1991)

17. Huang, L., Lian, X.R.: Regularity to the spherically symmetric compressible Navier-Stokes equations with density-dependent viscosity. Bound. Value Probl. 2018, 85 (2018)

18. Jiang, S., Xin, Z.P., Zhang, P.: Global weak solutions to 1D compressible isentropy Navier-Stokes with density-dependent viscosity. Methods Appl. Anal. 12(3), 239-252 (2005)

19. Kazhikhov, A.V., Shelukhin, V.V.: Unique global solution with respect to time of initial-boundary value problems for one-dimensional equations of a viscous gas. J. Appl. Math. Mech. 41, 273-282 (1977)

20. Li, J., Xin, Z.P.: Vanishing of vacuum states and blow-up phenomena of the compressible Navier-Stokes equations. Commun. Math. Phys. 281, 401-444 (2008)

21. Lian, R., Guo, Z.H., Li, H.L.: Dynamical behavior of vacuum states for 1D compressible Navier-Stokes equations. J. Differ. Equ. 248(8), 273-282 (2010)

22. Lions, P.L.: Mathematical Topics in Fluid Mechanics. Vol. 2. Compressible Models. Oxford University Press, New York (1998)

23. Liu, T.P., Xin, Z.P., Yang, T.: Vacuum states of compressible flow. Discrete Contin. Dyn. Syst. 4(1), 1-32 (1998)

24. Matsumura, A., Nishida, T.: The initial value problem for the equations of motion of compressible viscous and heat-conductive fluids. Proc. Jpn. Acad., Ser. A, Math. Sci. 55, 337-342 (1979)

25. Matsumura, A., Nishida, T.: The initial value problem for the equations of motion of viscous and heat-conductive gases. J. Math. Kyoto Univ. 20, 67-104 (1980)

26. Matsumura, A., Nishida, T.: The initial boundary value problems for the equations of motion of compressible and heat-conductive fluids. Commun. Math. Phys. 89, 445-464 (1983)

27. Mellet, A., Vasseur, A.: On the isentropic compressible Navier-Stokes equation. Commun. Partial Differ. Equ. 32(3), 431-452 (2007)

28. Nash, J.: Le probl'eme de Cauchy pour les equations differentielles dun fluide general. Bull. Soc. Math. Fr. 90, 487-497 (1962)

29. Serre, D.: Solutions faibles globales des equations de Navier-Stokes pourun fluide compressible. C. R. Acad. Sci. Paris, Ser. | 303, 639-642 (1986)

30. Serre, D.: Sur lquation monodimensionnelle dun fluide visqueux, compressible et conducteur de chaleur. C. R. Acad Sci. Paris, Ser. I 303(14), 703-706 (1986)

31. Serrin, J.: On the uniqueness of compressible fluid motion. Arch. Ration. Mech. Anal. 3, 271-288 (1959)

32. Shao, G.M., Chai, X.J.: Approximation of the $2 \mathrm{D}$ incompressible electrohydrodynamics system by the artificial compressibility method. Bound. Value Probl. 2017, 14 (2017) 
33. Tan, Z., Wang, Y.J., Wang, Y.: Stability of steady states of the Navier-Stokes-Poisson equations with non-flat doping profile. SIAM J. Math. Anal. 47(1), 179-209 (2015)

34. Vasseur, A., Yu, C.: Existence of global weak solutions for 3D degenerate compressible Navier-Stokes equations. Invent. Math. 206(3), 935-974 (2016)

35. Wang, Y.Z., Xiao, J.: Well/ill-posedness for the dissipative Navier-Stokes system in generalized Carleson measure spaces. Adv. Nonlinear Anal. 8(1), 203-224 (2019)

36. Xin, Z.P.: Blow-up of smooth solution to the compressible Navier-Stokes equations with compact density. Commun. Pure Appl. Math. 51, 229-240 (1998)

37. Xin, Z.P., Yuan, H.: Vacuum state for spherically symmetric solutions of the compressible Navier-Stokes equations. J. Hyperbolic Differ. Equ. 3, 403-442 (2006)

38. Zhang, T., Fang, D.Y.: Global behavior of spherically symmetric Navier-Stokes-Poisson system with degenerate viscosity coefficients. Arch. Ration. Mech. Anal. 191(2), 195-243 (2009)

39. Zhang, Y.H., Tan, Z:: On the existence of solutions to the Navier-Stokes-Poisson equations of a two-dimensional compressible flow. Math. Methods Appl. Sci. 30, 305-329 (2007)

40. Zlotnik, A.: On global in time properties of the symmetric compressible barotropic Navier-Stokes-Poisson flows in a vacuum. Int. Math. Ser. 7, 329-376 (2008)

\section{Submit your manuscript to a SpringerOpen ${ }^{0}$ journal and benefit from:}

- Convenient online submission

- Rigorous peer review

Open access: articles freely available online

- High visibility within the field

- Retaining the copyright to your article

Submit your next manuscript at $\gg$ springeropen.com 